\title{
A Class of Linear Codes with a Few Weights
}

\author{
Can Xiang ${ }^{1}$. Chunming Tang ${ }^{2}$. Keqin Feng ${ }^{3}$
}

Received: date / Accepted: date

\begin{abstract}
Linear codes have been an interesting subject of study for many years, as linear codes with few weights have applications in secrete sharing, authentication codes, association schemes, and strongly regular graphs. In this paper, a class of linear codes with a few weights over the finite field $\mathrm{GF}(p)$ are presented and their weight distributions are also determined, where $p$ is an odd prime. Some of the linear codes obtained are optimal in the sense that they meet certain bounds on linear codes.
\end{abstract}

Keywords Linear codes · weight distribution · weight enumerator $\cdot$ secret sharing schemes

Mathematics Subject Classification (2010) 94B05 · 94B15 · 94B60

\section{Introduction}

Throughout this paper, let $p$ be an odd prime and let $q=p^{m}$ for some positive integer $m$. An $[n, k, d]$ code $\mathcal{C}$ over $\operatorname{GF}(p)$ is a $k$-dimensional subspace of $\operatorname{GF}(p)^{n}$ with minimum (Hamming) distance $d$. Let $A_{i}$ denote the number of codewords with Hamming weight $i$ in a code $C$ of length $n$. The weight enumerator of $C$ is defined by $1+A_{1} z+A_{2} z^{2}+\cdots+A_{n} z^{n}$. The weight distribution $\left(1, A_{1}, \ldots, A_{n}\right)$ is an important research topic in coding theory, as it contains crucial information as to estimate the error correcting capability and the probability of error detection and correction with respect to some algorithms. A code $C$ is said to be a $t$-weight code if the number of nonzero $A_{i}$ in the sequence $\left(A_{1}, A_{2}, \cdots, A_{n}\right)$ is equal to $t$.

The research of K. Feng was supported by NSFC No. 11471178, 11571007 and the Tsinghua National Lab. for Information Science and Technology.

\section{Xiang}

College of Mathematics and Information Science, Guangzhou University, Guangzhou 510006, China

E-mail: cxiangcxiang@hotmail.com

C. Tang

School of Mathematics and Information, China West Normal University, Sichuan Nanchong, 637002, China

E-mail: tangchunmingmath@163.com

\section{K. Feng}

Department of Mathematical Sciences, Tsinghua University, Beijing, 100084, China

E-mail: kfeng@math.tsinghua.edu.cn 
Let $D=\left\{d_{1}, d_{2}, \ldots, d_{n}\right\} \subseteq \mathrm{GF}(q)$. Let $\operatorname{Tr}$ denote the trace function from $\operatorname{GF}(q)$ onto $\mathrm{GF}(p)$ throughout this paper. We define a linear code of length $n$ over $\mathrm{GF}(p)$ by

$$
\mathcal{C}_{D}=\left\{\left(\operatorname{Tr}\left(x d_{1}\right), \operatorname{Tr}\left(x d_{2}\right), \ldots, \operatorname{Tr}\left(x d_{n}\right)\right): x \in \mathrm{GF}(q)\right\}
$$

and call $D$ the defining set of this code $\mathcal{C}_{D}$. By definition, the dimension of the code $\mathcal{C}_{D}$ is at most $m$.

This construction is generic in the sense that many classes of known codes could be produced by properly selecting the defining set $D \subseteq \mathrm{GF}(q)$. If the defining set $D$ is well chosen, some optimal linear codes with few weights can be obtained. This construction technique was employed in [6], [13], [14], [7], [9], [10] and [25] for obtaining linear codes with a few weights. For more details, we refer interested readers to [8, 21, 29, 23, 24, 22] and the references therein.

The purpose of this paper is to construct a class of linear codes over $\operatorname{GF}(p)$ with a few nonzero weights using this generic construction method, and determine their weight distributions. Some of the linear codes obtained in this paper are optimal in the sense that they meet some bounds on linear codes. The linear codes with a few weights presented in this paper have applications also in secret sharing [1,4,27, authentication codes [11], combinatorial designs and graph theory [2],3], and association schemes [2], in addition to their applications in consumer electronics, communication and data storage systems.

The remainder of this paper is organized as follows. Section 2 introduces some basic notations and results of group characters, Gauss sums, exponential sums and cyclotomic fields which will be needed in subsequent sections. Section 3 presents a class of linear codes with a few weights and the proofs of their parameters are given in Section 4 Section 5 summarizes this paper.

\section{Preliminaries}

In this section, we state some notations and basic facts on group characters, Gauss sums, exponential sums and cyclotomic fields. These results will be used later in this paper.

\subsection{Some notations fixed throughout this paper}

For convenience, we adopt the following notations unless otherwise stated in this paper.

- $p^{*}=(-1)^{(p-1) / 2} p$.

- $\zeta_{p}=e^{\frac{2 \pi \sqrt{-1}}{p}}$, a primitive $p$-th root of unity.

- $L(x)=x^{p^{2}}+x$.

- $\operatorname{Im}(L)=\{L(x): x \in \mathrm{GF}(q)\}$.

- $x_{b} \in \operatorname{GF}(q)$ denotes a solution of the equation $L(x)=-b^{p}$ if $b \in \operatorname{Im}(L)$.

- $m_{p}=m$ mod $p \in\{0,1, \ldots, p-1\}$, the least non-negative residue modulo $m$.

- SQ and NSQ denote the set of all squares and nonsquares in $\operatorname{GF}(p)^{*}$, respectively.

- $\eta$ and $\bar{\eta}$ are the quadratic characters of $\operatorname{GF}(q)^{*}$ and $\operatorname{GF}(p)^{*}$, repsectively. We extend these quadratic characters by letting $\eta(0)=0$ and $\bar{\eta}(0)=0$. 
2.2 Group characters and Gauss sums

An additive character of $\operatorname{GF}(q)$ is a nonzero function $\chi$ from $\operatorname{GF}(q)$ to the set of nonzero complex numbers such that $\chi(x+y)=\chi(x) \chi(y)$ for any pair $(x, y) \in \operatorname{GF}(q)^{2}$. For each $b \in$ $\mathrm{GF}(q)$, the function

$$
\chi_{b}(c)=\zeta_{p}^{\operatorname{Tr}(b c)} \text { for all } c \in \mathrm{GF}(q)
$$

defines an additive character of $\operatorname{GF}(q)$. When $b=0, \chi_{0}(c)=1$ for all $c \in \operatorname{GF}(q)$, and is called the trivial additive character of $\operatorname{GF}(q)$. The character $\chi_{1}$ in (2) is called the canonical additive character of $\operatorname{GF}(q)$. It is known that every additive character of $\operatorname{GF}(q)$ can be written as $\chi_{b}(x)=\chi_{1}(b x)$ [20, Theorem 5.7].

The Gauss sum $G\left(\eta, \chi_{1}\right)$ over $\operatorname{GF}(q)$ is defined by

$$
G\left(\eta, \chi_{1}\right)=\sum_{c \in \mathrm{GF}(q)^{*}} \eta(c) \chi_{1}(c)=\sum_{c \in \mathrm{GF}(q)} \eta(c) \chi_{1}(c)
$$

and the Gauss sum $G\left(\bar{\eta}, \bar{\chi}_{1}\right)$ over $\mathrm{GF}(p)$ is defined by

$$
G\left(\bar{\eta}, \bar{\chi}_{1}\right)=\sum_{c \in \operatorname{GF}(p)^{*}} \bar{\eta}(c) \bar{\chi}_{1}(c)=\sum_{c \in \operatorname{GF}(p)} \bar{\eta}(c) \bar{\chi}_{1}(c),
$$

where $\bar{\chi}_{1}$ is the canonical additive characters of $\mathrm{GF}(p)$.

The following three lemmas are proved in [20, Theorem 5.15 and Theorem 5.33] and [14, lemma 7], respectively.

Lemma 1 With the symbols and notations above, we have

$$
G\left(\eta, \chi_{1}\right)=(-1)^{m-1} \sqrt{-1}^{\left(\frac{p-1}{2}\right)^{2} m} \sqrt{q}
$$

and

$$
G\left(\bar{\eta}, \bar{\chi}_{1}\right)=\sqrt{-1}^{\left(\frac{p-1}{2}\right)^{2}} \sqrt{p}=\sqrt{p *} .
$$

Lemma 2 Let $\chi$ be a nontrivial additive character of $\mathrm{GF}(q)$ with $q$ odd, and let $f(x)=$ $a_{2} x^{2}+a_{1} x+a_{0} \in \mathrm{GF}(q)[x]$ with $a_{2} \neq 0$. Then

$$
\sum_{c \in \mathrm{GF}(q)} \chi(f(c))=\chi\left(a_{0}-a_{1}^{2}\left(4 a_{2}\right)^{-1}\right) \eta\left(a_{2}\right) G(\eta, \chi) .
$$

Lemma 3 If $m \geq 2$ is even, then $\eta(y)=1$ for each $y \in \operatorname{GF}(p)^{*}$. If $m \geq 1$ is odd, then $\eta(y)=\bar{\eta}(y)$ for each $y \in \mathrm{GF}(p)$.

\subsection{A type of exponential sums}

For any $a$ and $b$ in $\operatorname{GF}(q)$, we define the following exponential sum

$$
S(a, b)=\sum_{x \in \mathrm{GF}(q)} \chi_{1}\left(a x^{p+1}+b x\right)
$$

in this paper. To prove our main results, we need the values of the sum $S(a, b)$ and the help of a number of lemmas that are proved in [5, Theorem 1, Theorem 2, Theorem 2.3]. 
Lemma 4 Let $m$ be odd, $f(x)=a^{p} x^{p^{2}}+a x \in F_{q}[x]$ and $b \in \mathrm{GF}(q)$. Then $f(x)$ is a permutation polynomial over $\mathrm{GF}(q)$ and

$$
S(a, b)={\sqrt{p^{*}}}^{m} \eta(a) \chi_{1}\left(-a x_{a, b}^{p+1}\right),
$$

where $p *=(-1)^{\frac{p-1}{2}} p$ and $x_{a, b}$ is the unique solution of the equation $f(x)=-b^{p}$.

Particularly, $S(a, 0)={\sqrt{p^{*}}}^{m} \eta(a)$.

Lemma 5 Let $m$ be even, $f(x)=a^{p} x^{p^{2}}+a x \in F_{q}[x]$ and $b \in \mathrm{GF}(q)$. There are two cases.

1. If $a^{\frac{q-1}{p+1}} \neq(-1)^{\frac{m}{2}}$, then $f(x)$ is a permutation polynomial over $\operatorname{GF}(q)$. Let $x_{a, b}$ be the unique solution of the equation $f(x)=-b^{p}$. Then

$$
S(a, b)=(-1)^{\frac{m}{2}} p^{m / 2} \chi_{1}\left(-a x_{a, b}^{p+1}\right)
$$

2. If $a^{\frac{q-1}{p+1}}=(-1)^{\frac{m}{2}}$, then $f(x)$ is not a permutation polynomial over $\operatorname{GF}(q)$. We have $S(a, b)=0$ unless the the equation $f(x)=-b^{p}$ is solvable. If this equation is solvable, with solution $x_{a, b}$ say, then

$$
S(a, b)=-(-1)^{\frac{m}{2}} p^{m / 2+1} \chi_{1}\left(-a x_{a, b}^{p+1}\right) .
$$

Particularly,

$$
S(a, 0)= \begin{cases}(-1)^{\frac{m}{2}} p^{\frac{m}{2}} & \text { if } a^{\frac{q-1}{p+1}} \neq(-1)^{\frac{m}{2}} \\ (-1)^{\frac{m}{2}+1} p^{\frac{m}{2}+1} & \text { if } a^{\frac{q-1}{p+1}}=(-1)^{\frac{m}{2}}\end{cases}
$$

2.4 Cyclotomic fields

In this subsection, we state some basic facts on cyclotomic fields. These results will be used in the rest of this paper.

Let $\mathbb{Z}$ be the rational integer ring and $Q$ be the rational field. Some results on cyclotomic field $Q\left(\zeta_{p}\right)[17]$ are given in the following lemma.

Lemma 6 We have the following basic facts.

1. The ring of integers in $K=Q\left(\zeta_{p}\right)$ is $O_{K}=\mathbb{Z}\left[\zeta_{p}\right]$ and $\left\{\zeta_{p}{ }^{i}: 1 \leq i \leq p-1\right\}$ is an integral basis of $O_{K}$.

2. The field extension $K / Q$ is Galois of degree $p-1$ and the Galois $\operatorname{group} \operatorname{Gal}(K / Q)=$ $\left\{\sigma_{a}: a \in(\mathbb{Z} / p \mathbb{Z})^{*}\right\}$, where the automorphism $\sigma_{a}$ of $K$ is defined by $\sigma_{a}\left(\zeta_{p}\right)=\zeta_{p}^{a}$.

3. The field $K$ has a unique quadratic subfield $L=Q\left(\sqrt{p^{*}}\right)$. For $1 \leq a \leq p-1, \sigma_{a}\left(\sqrt{p^{*}}\right)=$ $\bar{\eta}(a) \sqrt{p^{*}}$. Therefore, the Galois group $\operatorname{Gal}(L / Q)$ is $\left\{1, \sigma_{\gamma}\right\}$, where $\gamma$ is any quadratic nonresidue in $\mathrm{GF}(p)$. 


\section{The linear codes with a few weights}

We only describe the codes and introduce their parameters in this section. The proofs of their parameters will be given in Section 4

In this paper, the defining set $D$ of the $\operatorname{code} \mathcal{C}_{D}$ of 10 is given by

$$
D=\left\{x \in \operatorname{GF}(q)^{*}: \operatorname{Tr}\left(x^{p+1}-x\right)=0\right\} .
$$

When $p=2$, the weight distribution of the code $\mathcal{C}_{D}$ was settled in [26]. In this paper, we study the code $\mathcal{C}_{D}$ for $p$ being an odd prime.

The following three theorems are the main results of this paper.

Theorem 1 Let $m \geq 3$ be odd, and let $D$ be defined in (5). Then the set $\mathcal{C}_{D}$ of (1) is an $[n, m]$ linear code over $\mathrm{GF}(p)$ with at most five weights and the weight distribution in Tables $\square$ and 2 where

$$
n=p^{m-1}-1+(-1)^{\frac{p-1}{2} \frac{m-1}{2}} \bar{\eta}\left(m_{p}\right) p^{\frac{m-1}{2}} .
$$

Table 1: The weight distribution of $\mathcal{C}_{D}$ of Theorem 1 when $m_{p}=0$

\begin{tabular}{|c|c|}
\hline Weight $w$ & Multiplicity $A_{w}$ \\
\hline 0 & 1 \\
\hline$(p-1) p^{m-2}$ & $p^{m-1}-1$ \\
\hline$(p-1)\left(p^{m-2}-(-1)^{\frac{p-1}{2} \frac{m-1}{2}} p^{\frac{m-3}{2}}\right)$ & $\frac{p-1}{2}\left(p^{m-2}+(-1)^{\frac{p-1}{2} \frac{m-1}{2}} p^{\frac{m-1}{2}}\right)$ \\
\hline$(p-1)\left(p^{m-2}+(-1)^{\frac{p-1}{2} \frac{m-1}{2}} p^{\frac{m-3}{2}}\right)$ & $\frac{p-1}{2}\left(p^{m-2}-(-1)^{\frac{p-1}{2} \frac{m-1}{2}} p^{\frac{m-1}{2}}\right)$ \\
\hline$(p-1) p^{m-2}+(-1)^{\frac{p-1}{2}} \frac{m-1}{2} p^{\frac{m-3}{2}}$ & $\frac{1}{2}(p-1)^{2} p^{m-2}$ \\
\hline$(p-1) p^{m-2}-(-1)^{\frac{p-1}{2}} \frac{m-1}{2} p^{\frac{m-3}{2}}$ & $\frac{1}{2}(p-1)^{2} p^{m-2}$ \\
\hline
\end{tabular}

Table 2: The weight distribution of $\mathcal{C}_{D}$ of Theorem 1 when $m_{p} \neq 0$

\begin{tabular}{|c|c|}
\hline Weight $w$ & Multiplicity $A_{w}$ \\
\hline 0 & 1 \\
\hline$(p-1) p^{m-2}$ & $p^{m-2}-1+\bar{\eta}\left(m_{p}\right)(-1)^{\frac{p-1}{2} \frac{m-1}{2}}(p-1) p^{\frac{m-3}{2}}$ \\
\hline$(p-1) p^{m-2}+\bar{\eta}\left(m_{p}\right)(-1)^{\frac{p-1}{2} \frac{m-1}{2}} p^{\frac{m-1}{2}}$ & $p^{m-2}(p-1)-\bar{\eta}\left(m_{p}\right)(-1)^{\frac{p-1}{2} \frac{m-1}{2}}(p-1) p^{\frac{m-3}{2}}$ \\
\hline$(p-1) p^{m-2}+\bar{\eta}\left(m_{p}\right)(-1)^{\frac{p-1}{2} \frac{m-1}{2}}(p+1) p^{\frac{m-3}{2}}$ & $\frac{1}{2}(p-1)(p-2) p^{\frac{m-3}{2}}\left(p^{\frac{m-1}{2}}-\bar{\eta}\left(m_{p}\right)(-1)^{\frac{p-1}{2}} \frac{m-1}{2}\right)$ \\
\hline$(p-1) p^{m-2}+\bar{\eta}\left(m_{p}\right)(-1)^{\frac{p-1}{2}} \frac{m-1}{2}(p-1) p^{\frac{m-3}{2}}$ & $\frac{p-1}{2}\left(p^{m-1}-\bar{\eta}\left(m_{p}\right)(-1)^{\frac{p-1}{2} \frac{m-1}{2}} p^{\frac{m-1}{2}}\right)$ \\
\hline$(p-1) p^{m-2}+\bar{\eta}\left(m_{p}\right)(-1)^{\frac{p-1}{2}} \frac{m-1}{2} p^{\frac{m-3}{2}}$ & $(p-1) p^{m-2}+\bar{\eta}\left(m_{p}\right)(-1)^{\frac{p-1}{2} \frac{m-1}{2}}(p-1)^{2} p^{\frac{m-3}{2}}$ \\
\hline
\end{tabular}

Example 1 Let $(p, m)=(3,5)$. Then the code $\mathcal{C}_{D}$ has parameters $[71,5,42]$ and weight enumerator $1+30 z^{42}+60 z^{45}+90 z^{48}+42 z^{51}+20 z^{54}$, which is verified by a Magma program.

Example 2 Let $(p, m)=(3,9)$. Then the code $\mathcal{C}_{D}$ has parameters $[6560,9,4320]$ and weight enumerator $1+2268 z^{4320}+4374 z^{4347}+6560 z^{4374}+4374 z^{4401}+2106 z^{4428}$, which is verified by a Magma program. 
Remark 1 The code $C_{D}$ of Theorem 1 is a five-weight linear code except in the following two cases:

1. When $m=3$ and $m_{p}=0$, the frequency of the weight $(p-1)\left(p^{m-2}-(-1)^{\frac{p-1}{2} \frac{m-1}{2}} p^{\frac{m-3}{2}}\right)$ of Table 1 turns out to be 0 . Thus, the code $\mathcal{C}_{D}$ has four nonzero weights.

2. When $m=3, m_{p} \neq 0$ and $p \equiv 2(\bmod 3)$, the frequency of the weight $(p-1) p^{m-2}$ of Table 2 turns out to be 0 . Thus, the code $\mathcal{C}_{D}$ has only four nonzero weights.

Example 3 Let $(p, m)=(3,3)$. Then the code $\mathcal{C}_{D}$ has parameters $[8,3,4]$ and weight enumerator $1+6 z^{4}+6 z^{5}+8 z^{6}+6 z^{7}$, which is verified by a Magma program. This code is almost optimal, since the optimal linear code has parameters $[8,3,5]$.

Example 4 Let $(p, m)=(5,3)$. Then the code $\mathcal{C}_{D}$ has parameters $[19,3,14]$ and weight enumerator $1+36 z^{14}+24 z^{15}+60 z^{16}+4 z^{19}$, which is verified by a Magma program. This code is optimal.

Theorem 2 Let $m \geq 2$ be even and $m \equiv 2(\bmod 4)$, and let $D$ be defined in (5). Then the set $\mathcal{C}_{D}$ of $(1)$ is an $[n, m]$ linear code over $\mathrm{GF}(p)$ with at most three weights and the weight distribution in Table 3 and Table 4 where

$$
n= \begin{cases}p^{m-1}-1-(p-1) p^{\frac{1}{2}\left(m-1+(-1)^{\frac{m}{2}}\right)} & \text { if } m_{p}=0, \\ p^{m-1}-1+p^{\frac{1}{2}\left(m-1+(-1)^{\frac{m}{2}}\right)} & \text { if } m_{p} \neq 0 .\end{cases}
$$

Table 3: The weight distribution of $\mathcal{C}_{D}$ of Theorem 2 when $m_{p}=0$

\begin{tabular}{|c|c|}
\hline Weight $w$ & Multiplicity $A_{w}$ \\
\hline 0 & 1 \\
\hline$(p-1) p^{m-2}$ & $p^{m-2}-(p-1) p^{\frac{m}{2}-1}-1$ \\
\hline$(p-1)\left(p^{m-2}-p^{\frac{m}{2}-1}\right)$ & $(p-1)\left(2 p^{m-2}+p^{\frac{m}{2}-1}\right)$ \\
\hline$(p-1) p^{m-2}-(p-2) p^{\frac{m}{2}-1}$ & $(p-1)^{2} p^{m-2}$ \\
\hline
\end{tabular}

Table 4: The weight distribution of $\mathcal{C}_{D}$ of Theorem 2 when $m_{p} \neq 0$

\begin{tabular}{|c|c|}
\hline Weight $w$ & Multiplicity $A_{w}$ \\
\hline 0 & 1 \\
\hline$(p-1) p^{m-2}$ & $p^{m-2}+\frac{p-1}{2}\left(p^{m-1}+p^{\frac{m}{2}}\right)-1$ \\
\hline$(p-1) p^{m-2}+p^{\frac{m}{2}-1}$ & $(p-1)\left(2 p^{m-2}-p^{\frac{m}{2}-1}\right)$ \\
\hline$(p-1) p^{m-2}+2 p^{\frac{m}{2}-1}$ & $\frac{(p-1)(p-2)}{2}\left(p^{m-2}-p^{\frac{m}{2}-1}\right)$ \\
\hline
\end{tabular}

Example 5 Let $(p, m)=(3,6)$. Then the code $\mathcal{C}_{D}$ has parameters $[224,6,144]$ and weight enumerator $1+342 z^{144}+324 z^{153}+62 z^{162}$, which is verified by a Magma program.

Example 6 Let $(p, m)=(5,6)$. Then the code $\mathcal{C}_{D}$ has parameters $[3149,6,2500]$ and weight enumerator $1+7124 z^{2500}+2525 z^{4900}+2550 z^{3600}$, which is verified by a Magma program. 
Remark 2 The code $\mathcal{C}_{D}$ of Theorem 2 has three weights except in the case $m=2$, as the frequency of the weight $(p-1) p^{m-2}+2 p^{\frac{m}{2}-1}$ of Table 4 turns out to be 0 . Hence, The code $C_{D}$ of Theorem 2 is a two-weight linear code if and only if $m=2$.

Example 7 Let $(p, m)=(3,2)$. Then the code $\mathcal{C}_{D}$ has parameters $[3,2,2]$ and weight enumerator $1+6 z^{2}+2 z^{3}$, which is verified by a Magma program. This code is optimal.

Theorem 3 Let $m \geq 6$ be even and $m \equiv 0(\bmod 4)$, and let $D$ be defined in (5). Then the set $\mathcal{C}_{D}$ of (11) is a four-weight linear code over $\mathrm{GF}(p)$ with the parameter $[n, m]$ and the weight distribution in Tables 5 and 6 where $n$ is defined by (7).

Table 5: The weight distribution of $\mathcal{C}_{D}$ of Theorem 3 when $m_{p}=0$

\begin{tabular}{|c|c|}
\hline Weight $w$ & Multiplicity $A_{w}$ \\
\hline 0 & 1 \\
\hline$p^{m-2}(p-1)-(p-1)^{2} p^{\frac{m}{2}-1}$ & $\left(p^{2}-1\right) p^{m-2}$ \\
\hline$p^{m-2}(p-1)$ & $p^{m-4}-(p-1) p^{\frac{m}{2}-2}-1$ \\
\hline$(p-1) p^{\frac{m}{2}}\left(p^{\frac{m}{2}-2}-1\right)$ & $(p-1)\left(2 p^{m-4}+p^{\frac{m}{2}-2}\right)$ \\
\hline$(p-1) p^{m-2}-(p-2) p^{\frac{m}{2}}$ & $(p-1)^{2} p^{m-4}$ \\
\hline
\end{tabular}

Table 6: The weight distribution of $\mathcal{C}_{D}$ of Theorem 3 when $m_{p} \neq 0$

\begin{tabular}{|c|c|}
\hline Weight $w$ & Multiplicity $A_{w}$ \\
\hline 0 & 1 \\
\hline$(p-1)\left(p^{\frac{m}{2}-1}+p^{m-2}\right)$ & $p^{m}-p^{m-2}$ \\
\hline$(p-1) p^{m-2}$ & $p^{m-4}+\frac{p-1}{2}\left(p^{\frac{m}{2}-1}+p^{m-3}\right)-1$ \\
\hline$(p-1) p^{m-2}+p^{\frac{m}{2}}$ & $(p-1)\left(2 p^{m-4}-p^{\frac{m}{2}-2}\right)$ \\
\hline$(p-1) p^{m-2}+2 p^{\frac{m}{2}}$ & $\frac{1}{2}(p-1)(p-2)\left(p^{m-4}-p^{\frac{m}{2}-2}\right)$ \\
\hline
\end{tabular}

Example 8 Let $(p, m)=(3,8)$. Then the code $\mathcal{C}_{D}$ has parameters $[2267,8,1458]$ and weight enumerator $1+350 z^{1458}+5832 z^{1512}+306 z^{1539}+32 z^{1620}$, which is verified by a Magma program.

Example 9 Let $(p, m)=(5,8)$. Then the code $\mathcal{C}_{D}$ has parameters $[78749,8,62500]$ and weight enumerator $1+7124 z^{62500}+375000 z^{63000}+4900 z^{63125}+3600 z^{63750}$, which is verified by a Magma program.

\section{The proofs of the main results}

Our task of this section is to prove Theorems 1, 2 and 3 respectively. To this end, we shall prove a few more auxiliary results before proving the main results of this paper. 
4.1 Some auxiliary results

Lemma 7 With the symbols and notations above, we have

$$
\sum_{c \in \mathrm{GF}(p)^{*}} S(c,-c)= \begin{cases}(-1)^{\frac{p-1}{2}} \bar{\eta}\left(m_{p}\right) \sqrt{p^{*}}+1 & \text { if } m \text { is odd, } \\ -(p-1) p^{\frac{1}{2}\left(m+1+(-1)^{\frac{m}{2}}\right)} & \text { if } m \text { is even and } m_{p}=0, \\ p^{\frac{1}{2}\left(m+1+(-1)^{\frac{m}{2}}\right)} & \text { if } m \text { is even and } m_{p} \neq 0 .\end{cases}
$$

Proof For any $c \in \mathrm{GF}(p)^{*}$, it is easily seen that $f(x)=c^{p} x^{p^{2}}+c x=-(-c)^{p}$ is solvable and $\frac{1}{2}$ is its solution.

By definition, Lemmas 4 and 5 , we have

$$
\begin{aligned}
& \sum_{c \in \operatorname{GF}(p)^{*}} S(c,-c) \\
& =\sum_{c \in \operatorname{GF}(p)^{*} x \in \operatorname{GF}(q)} \chi_{1}\left(c x^{p+1}-c x\right) \\
& = \begin{cases}\sum_{c \in \operatorname{GF}(p)^{*}} \sqrt{p^{*}} \eta(c) \chi_{1}\left(-\frac{c}{4}\right) & \text { if } m \text { is odd, } \\
\sum_{c \in \operatorname{GF}(p)^{*}}(-1)^{\frac{m}{2}} p^{m / 2} \chi_{1}\left(-\frac{c}{4}\right) & \text { if } m \equiv 2(\bmod 4), \\
\sum_{c \in \operatorname{GF}(p)^{*}}-(-1)^{\frac{m}{2}} p^{m / 2+1} \chi_{1}\left(-\frac{c}{4}\right) & \text { if } m \equiv 0(\bmod 4),\end{cases} \\
& = \begin{cases}\sqrt{p^{*}} \sum_{c \in \operatorname{GF}(p)^{*}} \bar{\eta}(c) \bar{\chi}_{1}\left(-\frac{c \operatorname{Tr}(1)}{4}\right) & \text { if } m \text { is odd, } \\
-p^{m / 2} \sum_{c \in \operatorname{GF}(p)^{*}} \bar{\chi}_{1}\left(\frac{c \operatorname{Tr}(1)}{4}\right) & \text { if } m \equiv 2(\bmod 4), \\
-p^{m / 2+1} \sum_{c \in \operatorname{GF}(p)^{*}} \bar{\chi}_{1}\left(\frac{c \operatorname{Tr}(1)}{4}\right) & \text { if } m \equiv 0(\bmod 4),\end{cases} \\
& =\left\{\begin{array}{ll}
\sqrt{p^{*}} \sum_{c \in \operatorname{GF}(p)^{*}} \bar{\eta}(c) & \text { if } m \text { is odd and } m_{p}=0, \\
\sqrt{p^{*}} \sum_{c \in \operatorname{GF}(p) *}\left(-\frac{m_{p}}{4}\right) \bar{\eta}\left(-\frac{c m_{p}}{4}\right) \bar{\chi}_{1}\left(-\frac{c m_{p}}{4}\right) & \text { if } m \text { is odd and } m_{p} \neq 0, \\
-p^{m / 2}(p-1) & \text { if } m \equiv 2(\bmod 4) \text { and } m_{p}=0, \\
p^{m / 2} & \text { if } m \equiv 2(\bmod 4) \text { and } m_{p} \neq 0, \\
-p^{m / 2+1}(p-1) & \text { if } m \equiv 0(\bmod 4) \text { and } m_{p}=0, \\
p^{m / 2+1} & \text { if } m \text { is odd and } m_{p}=0,
\end{array},\right. \\
& = \begin{cases}0 & \text { if } m \text { is odd and } m_{p} \neq 0, \\
\sqrt{p^{*}} \bar{\eta}\left(-m_{p}\right) G\left(\bar{\eta}, \bar{\chi}_{1}\right) \\
-p^{m / 2}(p-1) & \text { if } m \equiv 2(\bmod 4) \text { and } m_{p}=0, \\
p^{m / 2} & \text { if } m \equiv 0(\bmod 4) \text { and } m_{p} \neq 0, \\
-p^{m / 2+1}(p-1) & \text { and } m_{p}=0, \\
p^{m / 2+1} & \bmod 4) \text { and } m_{p} \neq 0 .\end{cases}
\end{aligned}
$$

The desired conclusion then follows from Lemma 1

The next lemma will be employed in proving the code length.

\section{Lemma 8 Let}

$$
n_{0}=\left|\left\{x \in \operatorname{GF}(q): \operatorname{Tr}\left(x^{p+1}-x\right)=0\right\}\right| .
$$

Then

$$
n_{0}= \begin{cases}p^{m-1}+(-1)^{\frac{p-1}{2}} \bar{\eta}\left(m_{p}\right) p^{-1}{\sqrt{p^{*}}}^{m+1} & \text { if } m \text { is odd, } \\ p^{m-1}-(p-1) p^{\frac{1}{2}\left(m-1+(-1)^{\frac{m}{2}}\right)} & \text { if } m \text { is even and } m_{p}=0, \\ p^{m-1}+p^{\frac{1}{2}\left(m-1+(-1)^{\frac{m}{2}}\right)} & \text { if } m \text { is even and } m_{p} \neq 0 .\end{cases}
$$


Proof By definition, we have

$$
\begin{aligned}
n_{0} & =\frac{1}{p} \sum_{x \in \mathrm{GF}(q)} \sum_{y \in \mathrm{GF}(p)} \zeta_{p}^{y\left(\operatorname{Tr}\left(x^{p+1}\right)-x\right)} \\
& =p^{m-1}+\frac{1}{p} \sum_{y \in \mathrm{GF}(p)^{*}} \sum_{x \in \mathrm{GF}(q)} \zeta_{p}^{\operatorname{Tr}\left(y x^{p+1}-y\right)} \\
& =p^{m-1}+\frac{1}{p} \sum_{y \in \mathrm{GF}(p)^{*}} S(y,-y)
\end{aligned}
$$

The desired conclusion then follows from Lemma 7

From Lemma 6 the conclusion of the following lemma is straightforward and we omit their proofs.

Lemma 9 With the symbols and notations above, we have the following.

1. $\sum_{y \in \mathrm{GF}(p)^{*}} \sigma_{y}\left(\zeta_{p}^{z}\right)= \begin{cases}p-1 & \text { if } z=0, \\ -1 & \text { if } z \neq 0 .\end{cases}$

2. $\sum_{y \in \operatorname{GF}(p)^{*}} \sigma_{y}\left({\sqrt{p^{*}}}^{m}\right)= \begin{cases}0 & \text { if } m \text { is odd }, \\ {\sqrt{p^{*}}}^{m}(p-1) & \text { if } m \text { is even. }\end{cases}$

3. $\sum_{y \in \mathrm{GF}(p)^{*}} \sigma_{y}\left(\sqrt{p^{*}} \zeta_{p}^{z}\right)= \begin{cases}0 & \text { if } z=0, \\ \bar{\eta}(z) p^{*} & \text { if } z \neq 0 .\end{cases}$

Particularly,

$$
\sum_{y \in \operatorname{GF}(p)^{*}} \sigma_{y}\left({\sqrt{p^{*}}}^{m} \zeta_{p}^{-\frac{m_{p}}{4}}\right)= \begin{cases}0 & \text { if } m \text { is odd and } m_{p}=0 \\ (-1)^{\frac{p-1}{2}} \bar{\eta}\left(m_{p}\right){\sqrt{p^{*}}}^{m+1} & \text { if } m \text { is odd and } m_{p} \neq 0 .\end{cases}
$$

and

$$
\sum_{y \in \operatorname{GF}(p)^{*}} \sigma_{y}\left(\zeta_{p}^{-\frac{m_{p}}{4}}\right)= \begin{cases}p-1 & \text { if } m_{p}=0 \\ -1 & \text { if } m_{p} \neq 0\end{cases}
$$

The following result will play an important role in proving the main results of this paper.

Lemma 10 Let $b \in \mathrm{GF}(q)^{*}, L(x)=x^{p^{2}}+x$ and

$$
M=\sum_{y \in \operatorname{GF}(p)^{*}} \sum_{z \in \operatorname{GF}(p)^{*}} \sum_{x \in \operatorname{GF}(q)} \zeta_{p}^{\operatorname{Tr}\left(y x^{p+1}+(b z-y) x\right)} .
$$

(I) If $m$ is odd, then we have the following.

- When $m_{p}=0$,

$$
M= \begin{cases}0 & \text { if } \operatorname{Tr}\left(x_{b}^{p+1}\right)=0, \\ (-1)^{\frac{p-1}{2}}(p-1){\sqrt{p^{*}}}^{m+1} & \text { if } \operatorname{Tr}\left(x_{b}^{p+1}\right) \in \mathrm{SQ} \text { and } \operatorname{Tr}\left(x_{b}\right)=0, \\ -(-1)^{\frac{p-1}{2}}(p-1){\sqrt{p^{*}}}^{m+1} & \text { if } \operatorname{Tr}\left(x_{b}^{p+1}\right) \in \mathrm{NSQ} \text { and } \operatorname{Tr}\left(x_{b}\right)=0, \\ -(-1)^{\frac{p-1}{2}}{\sqrt{p^{*}}}^{m+1} & \text { if } \operatorname{Tr}\left(x_{b}^{p+1}\right) \in \mathrm{SQ} \text { and } \operatorname{Tr}\left(x_{b}\right) \neq 0, \\ (-1)^{\frac{p-1}{2}}{\sqrt{p^{*}}}^{m+1} & \text { if } \operatorname{Tr}\left(x_{b}^{p+1}\right) \in \mathrm{NSQ} \text { and } \operatorname{Tr}\left(x_{b}\right) \neq 0 .\end{cases}
$$


- When $m_{p} \in \mathrm{SQ}$,

$$
M= \begin{cases}(-1)^{\frac{p-1}{2}}(p-1){\sqrt{p^{*}}}^{m+1} & \text { if } \operatorname{Tr}\left(x_{b}^{p+1}\right)=0 \text { and } \operatorname{Tr}\left(x_{b}\right)=0, \\ -(-1)^{\frac{p-1}{2}} \sqrt{p^{*}} & \text { if } \operatorname{Tr}\left(x_{b}^{p+1}\right)=0 \text { and } \operatorname{Tr}\left(x_{b}\right) \neq 0, \\ -2 \cdot(-1)^{\frac{p-1}{2}}{\sqrt{p^{*}}}^{m+1} & \text { if } \operatorname{Tr}\left(x_{b}^{p+1}\right) \in \mathrm{SQ} \text { and } \operatorname{Tr}\left(x_{b}\right)=0, \\ 0 & \text { if } \operatorname{Tr}\left(x_{b}^{p+1}\right) \in \mathrm{NSQ}, \\ (p-2)(-1)^{\frac{p-1}{2}}{\sqrt{p^{*}}}^{m+1} \text { or }-2 \cdot(-1)^{\frac{p-1}{2}}{\sqrt{p^{*}}}^{m+1} & \text { if } \operatorname{Tr}\left(x_{b}^{p+1}\right) \in \mathrm{SQ} \text { and } \operatorname{Tr}\left(x_{b}\right) \neq 0 .\end{cases}
$$

- When $m_{p} \in \mathrm{NSQ}$,

$$
M= \begin{cases}-(-1)^{\frac{p-1}{2}}(p-1){\sqrt{p^{*}}}^{m+1} & \text { if } \operatorname{Tr}\left(x_{b}^{p+1}\right)=0 \text { and } \operatorname{Tr}\left(x_{b}\right)=0, \\ (-1)^{\frac{p-1}{2}} \sqrt{p^{*}} & \text { if } \operatorname{Tr}\left(x_{b}^{p+1}\right)=0 \text { and } \operatorname{Tr}\left(x_{b}\right) \neq 0, \\ 0 & \text { if } \operatorname{Tr}\left(x_{b}^{p+1}\right) \in \operatorname{SQ}, \\ 2 \cdot(-1)^{\frac{p-1}{2}}{\sqrt{p^{*}}}^{m+1} & \text { if } \operatorname{Tr}\left(x_{b}^{p+1}\right) \in \mathrm{NSQ} \text { and } \operatorname{Tr}\left(x_{b}\right)=0, \\ -(p-2)(-1)^{\frac{p-1}{2}}{\sqrt{p^{*}}}^{m+1} \text { or } 2 \cdot(-1)^{\frac{p-1}{2}}{\sqrt{p^{*}}}^{m+1} & \text { if } \operatorname{Tr}\left(x_{b}^{p+1}\right) \in \mathrm{NSQ} \text { and } \operatorname{Tr}\left(x_{b}\right) \neq 0 .\end{cases}
$$

where $x_{b}$ is the unique solution of the equation $x^{p^{2}}+x=-b^{p}$.

(II) If $m \equiv 2(\bmod 4)$, then we have the following.

- When $m_{p}=0$,

$$
M= \begin{cases}-p^{\frac{m}{2}}(p-1)^{2} & \text { if } \operatorname{Tr}\left(x_{b}^{p+1}\right)=0 \text { and } \operatorname{Tr}\left(x_{b}\right)=0, \\ p^{\frac{m}{2}}(p-1) & \text { if } \operatorname{Tr}\left(x_{b}^{p+1}\right)=0 \text { and } \operatorname{Tr}\left(x_{b}\right) \neq 0 \\ & \text { or } \operatorname{Tr}\left(x_{b}^{p+1}\right) \neq 0 \text { and } \operatorname{Tr}\left(x_{b}\right)=0, \\ -p^{\frac{m}{2}} & \text { if } \operatorname{Tr}\left(x_{b}^{p+1}\right) \neq 0 \text { and } \operatorname{Tr}\left(x_{b}\right) \neq 0 .\end{cases}
$$

- When $m_{p} \neq 0$

$$
M= \begin{cases}p^{\frac{m}{2}}(p-1) & \text { if } \operatorname{Tr}\left(x_{b}^{p+1}\right)=0 \text { and } \operatorname{Tr}\left(x_{b}\right)=0, \\ -p^{\frac{m}{2}} & \text { if } \operatorname{Tr}\left(x_{b}^{p+1}\right)=0 \text { and } \operatorname{Tr}\left(x_{b}\right) \neq 0 \\ & \text { or } \operatorname{Tr}\left(x_{b}^{p+1}\right) \neq 0 \text { and } A=0, \\ p^{\frac{m}{2}}(p-1) \text { or }-p^{\frac{m}{2}}(p+1) & \text { if } \operatorname{Tr}\left(x_{b}^{p+1}\right) \neq 0 \text { and } A \neq 0,\end{cases}
$$

where $A=-\frac{m_{p}}{4}+\frac{\operatorname{Tr}\left(x_{b}\right)^{2}}{4 \operatorname{Tr}\left(x_{b}^{p+1}\right)}$ and $x_{b}$ is the unique solution of the equation $x^{p^{2}}+x=-b^{p}$.

(III) If $m \equiv 0(\bmod 4)$, then we have the following.

- When $m_{p}=0$,

$$
M= \begin{cases}0 & \text { if } b \notin \operatorname{Im}(L), \\ -p^{\frac{m}{2}+1}(p-1)^{2} & \text { if } b \in \operatorname{Im}(L), \operatorname{Tr}\left(x_{b}^{p+1}\right)=0 \text { and } \operatorname{Tr}\left(x_{b}\right)=0, \\ p^{\frac{m}{2}+1}(p-1) & \text { if } b \in \operatorname{Im}(L), \operatorname{Tr}\left(x_{b}^{p+1}\right)=0 \text { and } \operatorname{Tr}\left(x_{b}\right) \neq 0 \\ & \text { or } \operatorname{Tr}\left(x_{b}^{p+1}\right) \neq 0 \text { and } \operatorname{Tr}\left(x_{b}\right)=0 \\ -p^{\frac{m}{2}+1} & \text { if } b \in \operatorname{Im}(L), \operatorname{Tr}\left(x_{b}^{p+1}\right) \neq 0 \text { and } \operatorname{Tr}\left(x_{b}\right) \neq 0 .\end{cases}
$$

- When $m_{p} \neq 0$,

$$
M= \begin{cases}0 & \text { if } b \notin \operatorname{Im}(L), \\ p^{\frac{m}{2}+1}(p-1) & \text { if } b \in \operatorname{Im}(L), \operatorname{Tr}\left(x_{b}^{p+1}\right)=0 \text { and } \operatorname{Tr}\left(x_{b}\right)=0, \\ -p^{\frac{m}{2}+1} & \text { if } b \in \operatorname{Im}(L), \operatorname{Tr}\left(x_{b}^{p+1}\right)=0 \text { and } \operatorname{Tr}\left(x_{b}\right) \neq 0 \\ & \text { or } \operatorname{Tr}\left(x_{b}^{p+1}\right) \neq 0 \text { and } A=0, \\ p^{\frac{m}{2}+1}(p-1) \text { or }-p^{\frac{m}{2}+1}(p+1) & \text { if } b \in \operatorname{Im}(L), \operatorname{Tr}\left(x_{b}^{p+1}\right) \neq 0 \text { and } A \neq 0 .\end{cases}
$$


where $A=-\frac{m_{p}}{4}+\frac{\operatorname{Tr}\left(x_{b}\right)^{2}}{4 \operatorname{Tr}\left(x_{b}^{p+1}\right)}$ and $x_{b}$ is some solution of the equation $L(x)=-b^{p}$ under the condition that $b \in \operatorname{Im}(L)$.

Proof We have

$$
\begin{aligned}
M & =\sum_{y \in \mathrm{GF}(p)^{*}} \sum_{z \in \mathrm{GF}(p)^{*}} \sum_{x \in \mathrm{GF}(q)} \zeta_{p}^{\operatorname{Tr}\left(y x^{p+1}+(b z-y) x\right)} \\
& =\sum_{y \in \mathrm{GF}(p)^{*}} \sum_{z \in \mathrm{GF}(p)^{*}} \sum_{x \in \mathrm{GF}(q)} \zeta_{p}^{y \operatorname{Tr}\left(x^{p+1}+\left(\frac{z}{y} b-1\right) x\right)} \\
& =\sum_{y \in \mathrm{GF}(p)^{*}} \sum_{z \in \mathrm{GF}(p)^{*}} \sum_{x \in \mathrm{GF}(q)} \zeta_{p}^{y \operatorname{Tr}\left(x^{p+1}+(z b-1) x\right)} \\
& =\sum_{y \in \mathrm{GF}(p)^{*}} \sigma_{y}\left(\sum_{z \in \mathrm{GF}(p)^{*}} \sum_{x \in \mathrm{GF}(q)} \zeta_{p}^{\operatorname{Tr}\left(x^{p+1}+(b z-1) x\right)}\right) .
\end{aligned}
$$

It is easily seen that $L(x)=x^{p^{2}}+x$ is a permutation polynomial over $\operatorname{GF}(q)$ if $m \equiv 2$ $(\bmod 4)$ or $m$ is odd. Hence, $x_{b}$ is the unique solution of the equation $L(x)=-b^{p}$, while $z x_{b}+\frac{1}{2}$ is the unique solution of the equation $L(x)=-(b z-1)^{p}$ for $z \in \operatorname{GF}(p)^{*}$.

For $m \equiv 0(\bmod 4)$, although $L(x)=x^{p^{2}}+x$ is not a permutation polynomial over $\mathrm{GF}(q), z x_{b}+\frac{1}{2}$ is the solution of the equation $L(x)=-(b z-1)^{p}$ for $z \in \mathrm{GF}(p)^{*}$ when $b \in \operatorname{Im}(L)$ with some solution $x_{b}$.

Therefore, by Lemmas 4 and 5 , Equation (8) becomes

$$
\begin{aligned}
& M= \begin{cases}\sum_{y \in \operatorname{GF}(p)^{*}} \sigma_{y}\left(\sum_{z \in \operatorname{GF}(p)^{*}} \sqrt{p^{*}} \eta(1) \zeta_{p}^{-\operatorname{Tr}\left(\left(z x_{b}+\frac{1}{2}\right)^{p+1}\right)}\right) & \text { if } m \text { is odd } \\
\sum_{y \in \operatorname{GF}(p)^{*}} \sigma_{y}\left(\sum_{z \in \operatorname{GF}(p)^{*}}(-1)^{\frac{m}{2}} p^{\frac{m}{2}} \zeta_{p}^{-\operatorname{Tr}\left(\left(z x_{b}+\frac{1}{2}\right)^{p+1}\right)}\right) & \text { if } m \equiv 2(\bmod 4) \\
0 & \text { if } m \equiv 0(\bmod 4) \text { and } b \notin \operatorname{Im}(L) \\
\sum_{y \in \operatorname{GF}(p)^{*}} \sigma_{y}\left(\sum_{z \in \operatorname{GF}(p)^{*}}-(-1)^{\frac{m}{2}} p^{\frac{m}{2}+1} \zeta_{p}^{-\operatorname{Tr}\left(\left(z x_{b}+\frac{1}{2}\right)^{p+1}\right)}\right) & \text { if } m \equiv 0(\bmod 4) \text { and } b \in \operatorname{Im}(L)\end{cases}
\end{aligned}
$$

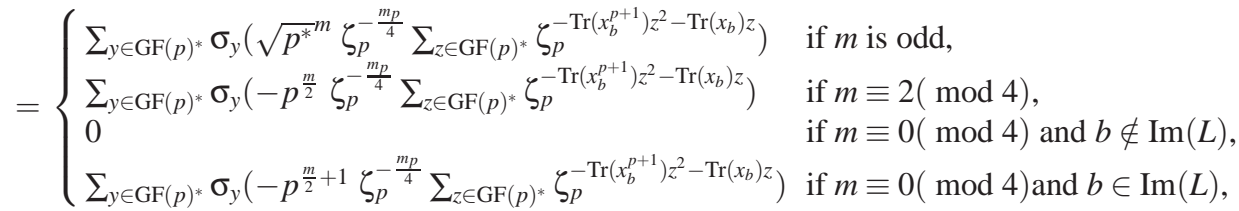

We distinguish the following three cases.

(I) If $m$ is odd, by Lemma2, we have

$$
\begin{aligned}
M= & \begin{cases}\sum_{y \in \operatorname{GF}(p)^{*}} \sigma_{y}\left({\sqrt{p^{*}}}^{m} \zeta_{p}^{-\frac{m_{p}}{4}} \sum_{z \in \operatorname{GF}(p)^{*}} \zeta_{p}^{-\operatorname{Tr}\left(x_{b}\right) z}\right) & \text { if } \operatorname{Tr}\left(x_{b}^{p+1}\right)=0 \\
\sum_{y \in \operatorname{GF}(p)^{*}} \sigma_{y}\left({\sqrt{p^{*}}}^{m} \zeta_{p}^{-\frac{m_{p}}{4}}\left(\bar{\eta}\left(-\operatorname{Tr}\left(x_{b}^{p+1}\right) \zeta_{p}^{\frac{\operatorname{Tr}\left(x_{b}^{p+1}\right)}{p^{p+1}}} G\left(\bar{\eta}, \bar{\chi}_{1}\right)-1\right)\right)\right. & \text { if } \operatorname{Tr}\left(x_{b}^{p+1}\right) \neq 0\end{cases} \\
= & \begin{array}{ll}
(p-1) A_{0} & \text { if } \operatorname{Tr}\left(x_{b}^{p+1}\right)=0 \text { and } \operatorname{Tr}\left(x_{b}\right)=0, \\
-A_{0} & \text { if } \operatorname{Tr}\left(x_{b}^{p+1}\right)=0 \text { and } \operatorname{Tr}\left(x_{b}\right) \neq 0, \\
{\sqrt{p^{*}}}^{m+1} \bar{\eta}\left(-\operatorname{Tr}\left(x_{b}^{p+1}\right) \sum_{y \in \operatorname{GF}(p)^{*}} \sigma_{y}\left(\zeta_{p}^{-\frac{m_{p}}{4}}\right)-A_{0}\right. & \text { if } \operatorname{Tr}\left(x_{b}^{p+1}\right) \neq 0 \text { and } \operatorname{Tr}\left(x_{b}\right)=0, \\
{\sqrt{p^{*}}}^{m+1} \bar{\eta}\left(-\operatorname{Tr}\left(x_{b}^{p+1}\right) \sum_{y \in \operatorname{GF}(p)^{*}} \sigma_{y}\left(\zeta_{p}^{A}\right)-A_{0}\right. & \text { if } \operatorname{Tr}\left(x_{b}^{p+1}\right) \neq 0 \text { and } \operatorname{Tr}\left(x_{b}\right) \neq 0,
\end{array}
\end{aligned}
$$

where $A_{0}=\sum_{y \in \operatorname{GF}(p)^{*}} \sigma_{y}\left({\sqrt{p^{*}}}^{m} \zeta_{p}^{-\frac{m_{p}}{4}}\right)$ and $A=-\frac{m_{p}}{4}+\frac{\operatorname{Tr}\left(x_{b}\right)^{2}}{4 \operatorname{Tr}\left(x_{b}^{p+1}\right)}$. The desired conclusion in Part (I) of this lemma then follows from Lemma 9 
(II) If $m \equiv 2(\bmod 4)$, by Lemmas 2 and 1 we have

$$
\begin{aligned}
& M= \begin{cases}\sum_{y \in \operatorname{GF}(p)^{*}} \sigma_{y}\left(-p^{\frac{m}{2}} \zeta_{p}^{-\frac{m_{p}}{4}} \sum_{z \in \operatorname{GF}(p)^{*}} \zeta_{p}^{-\operatorname{Tr}\left(x_{b}\right) z}\right) & \text { if } \operatorname{Tr}\left(x_{b}^{p+1}\right)=0 \\
\sum_{y \in \operatorname{GF}(p)^{*}} \sigma_{y}\left(-p^{\frac{m}{2}} \zeta_{p}^{-\frac{m_{p}}{4}}\left(\sum_{z \in \operatorname{GF}(p)} \zeta_{p}^{-\operatorname{Tr}\left(x_{b}^{p+1}\right) z^{2}-\operatorname{Tr}\left(x_{b}\right) z}-1\right)\right) & \text { if } \operatorname{Tr}\left(x_{b}^{p+1}\right) \neq 0\end{cases}
\end{aligned}
$$

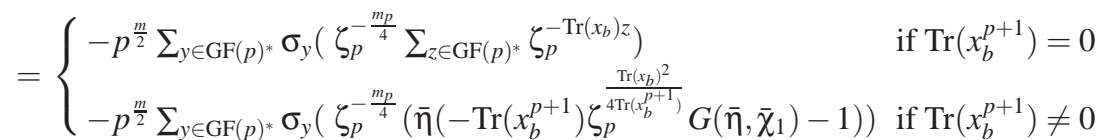

$$
\begin{aligned}
& = \begin{cases}-p^{\frac{m}{2}}(p-1) A_{1} & \text { if } \operatorname{Tr}\left(x_{b}^{p+1}\right)=0 \text { and } \operatorname{Tr}\left(x_{b}\right)=0, \\
p^{\frac{m}{2}} A_{1} & \text { if } \operatorname{Tr}\left(x_{b}^{p+1}\right)=0 \text { and } \operatorname{Tr}\left(x_{b}\right) \neq 0, \\
-p^{\frac{m}{2}}(-1)^{\frac{p-1}{2}} \bar{\eta}\left(\operatorname{Tr}\left(x_{b}^{p+1}\right)\right) \sum_{y \in \operatorname{GF}(p)^{*}} \sigma_{y}\left(\sqrt{p^{*} \zeta_{p}^{-\frac{m_{p}}{4}}}\right)+p^{\frac{m}{2}} A_{1} & \text { if } \operatorname{Tr}\left(x_{b}^{p+1}\right) \neq 0 \text { and } \operatorname{Tr}\left(x_{b}\right)=0, \\
-p^{\frac{m}{2}}(-1)^{\frac{p-1}{2}} \bar{\eta}\left(\operatorname{Tr}\left(x_{b}^{p+1}\right)\right) \sum_{y \in \operatorname{GF}(p)^{*}} \sigma_{y}\left(\sqrt{p^{*} \zeta_{p}}\right)+p^{\frac{m}{2}} A_{1} & \text { if } \operatorname{Tr}\left(x_{b}^{p+1}\right) \neq 0 \text { and } \operatorname{Tr}\left(x_{b}\right) \neq 0,\end{cases}
\end{aligned}
$$

where $A_{1}=\sum_{y \in \operatorname{GF}(p)^{*}} \sigma_{y}\left(\zeta_{p}^{-\frac{m_{p}}{4}}\right)$ and $A=-\frac{m_{p}}{4}+\frac{\operatorname{Tr}\left(x_{b}\right)^{2}}{4 \operatorname{Tr}\left(x_{b}^{p+1}\right)}$. The desired conclusion in Part (II) of this lemma then follows from Lemma 9 .

(III) If $m \equiv 0(\bmod 4)$, then from Lemmas 2 and 1 we have

$$
\begin{aligned}
M & = \begin{cases}0 & \text { if } b \notin \operatorname{Im}(L) \\
-p^{\frac{m}{2}+1} \sum_{y \in \operatorname{GF}(p)^{*}} \sigma_{y}\left(\zeta_{p}^{-\frac{m_{p}}{4}} \sum_{z \in \operatorname{GF}(p)^{*}} \zeta_{p}^{-\operatorname{Tr}\left(x_{b}\right) z}\right) & \text { if } b \in \operatorname{Im}(L) \text { and } \operatorname{Tr}\left(x_{b}^{p+1}\right)=0 \\
-p^{\frac{m}{2}+1} \sum_{y \in \operatorname{GF}(p)^{*}} \sigma_{y}\left(\zeta_{p}^{-\frac{m_{p}}{4}}\left(\sum_{z \in \operatorname{GF}(p)} \zeta_{p}^{-\operatorname{Tr}\left(x_{b}^{p+1}\right) z^{2}-\operatorname{Tr}\left(x_{b}\right) z}-1\right)\right) & \text { if } b \in \operatorname{Im}(L) \text { and } \operatorname{Tr}\left(x_{b}^{p+1}\right) \neq 0\end{cases} \\
& = \begin{cases}0 & \text { if } b \notin \operatorname{Im}(L), \\
-p^{\frac{m}{2}+1}(p-1) A_{1} & \text { if } b \in \operatorname{Im}(L), \operatorname{Tr}\left(x_{b}^{p+1}\right)=0 \text { and } \operatorname{Tr}\left(x_{b}\right)=0, \\
p^{\frac{m}{2}+1} A_{1} & \text { if } b \in \operatorname{Im}(L), \operatorname{Tr}\left(x_{b}^{p+1}\right)=0 \text { and } \operatorname{Tr}\left(x_{b}\right) \neq 0, \\
-p^{\frac{m}{2}+1}(-1)^{\frac{p-1}{2}} \bar{\eta}\left(\operatorname{Tr}\left(x_{b}^{p+1}\right)\right) A+p^{\frac{m}{2}+1} A_{1} & \text { if } b \in \operatorname{Im}(L) \text { and } \operatorname{Tr}\left(x_{b}^{p+1}\right) \neq 0,\end{cases}
\end{aligned}
$$

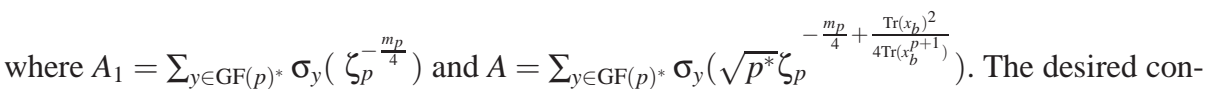
clusion in Part (III) of this lemma then follows from Lemma 9

This completes the proof of this lemma.

In order to calculate the Hamming weight of each codeword in $\mathcal{C}_{D}$, we need the following result.

Lemma 11 For any $b \in \mathrm{GF}(q)^{*}$, let

$$
N(b)=\mid\left\{x \in \mathrm{GF}(q): \operatorname{Tr}\left(x^{p+1}-x\right)=0 \text { and } \operatorname{Tr}(b x)=0\right\} \mid .
$$

There are three cases.

(I) If $m$ is odd, then we have the following.

- When $m_{p}=0$,

$$
N(b)= \begin{cases}p^{m-2} & \text { if } \operatorname{Tr}\left(x_{b}^{p+1}\right)=0, \\ (p-1) B+p^{m-2} & \text { if } \operatorname{Tr}\left(x_{b}^{p+1}\right) \in \mathrm{SQ} \text { and } \operatorname{Tr}\left(x_{b}\right)=0, \\ -(p-1) B+p^{m-2} & \text { if } \operatorname{Tr}\left(x_{b}^{p+1}\right) \in \mathrm{NSQ} \text { and } \operatorname{Tr}\left(x_{b}\right)=0, \\ -B+p^{m-2} & \text { if } \operatorname{Tr}\left(x_{b}^{p+1}\right) \in \mathrm{SQ} \text { and } \operatorname{Tr}\left(x_{b}\right) \neq 0, \\ B+p^{m-2} & \text { if } \operatorname{Tr}\left(x_{b}^{p+1}\right) \in \mathrm{NSQ} \text { and } \operatorname{Tr}\left(x_{b}\right) \neq 0 .\end{cases}
$$


- When $\bar{\eta}\left(m_{p}\right)=1$,

$$
N(b)= \begin{cases}p B+p^{m-2} & \text { if } \operatorname{Tr}\left(x_{b}^{p+1}\right)=0 \text { and } \operatorname{Tr}\left(x_{b}\right)=0, \\ p^{m-2} & \text { if } \operatorname{Tr}\left(x_{b}^{p+1}\right)=0 \text { and } \operatorname{Tr}\left(x_{b}\right) \neq 0, \\ -B+p^{m-2} & \text { if } \operatorname{Tr}\left(x_{b}^{p+1}\right) \in \mathrm{SQ} \text { and } \operatorname{Tr}\left(x_{b}\right)=0, \\ B+p^{m-2} & \text { if } \operatorname{Tr}\left(x_{b}^{p+1}\right) \in \mathrm{NSQ}, \\ (p-1) B+p^{m-2} \text { or }-B+p^{m-2} & \text { if } \operatorname{Tr}\left(x_{b}^{p+1}\right) \in \mathrm{SQ} \text { and } \operatorname{Tr}\left(x_{b}\right) \neq 0 .\end{cases}
$$

- When $\bar{\eta}\left(m_{p}\right)=-1$,

$$
N(b)= \begin{cases}-p B+p^{m-2} & \text { if } \operatorname{Tr}\left(x_{b}^{p+1}\right)=0 \text { and } \operatorname{Tr}\left(x_{b}\right)=0, \\ p^{m-2} & \text { if } \operatorname{Tr}\left(x_{b}^{p+1}\right)=0 \text { and } \operatorname{Tr}\left(x_{b}\right) \neq 0, \\ -B+p^{m-2} & \text { if } \operatorname{Tr}\left(x_{b}^{p+1}\right) \in \mathrm{SQ}, \\ B+p^{m-2} & \text { if } \operatorname{Tr}\left(x_{b}^{p+1}\right) \in \mathrm{NSQ} \text { and } \operatorname{Tr}\left(x_{b}\right)=0, \\ -(p-1) B+p^{m-2} \text { or } B+p^{m-2} & \text { if } \operatorname{Tr}\left(x_{b}^{p+1}\right) \in \mathrm{NSQ} \text { and } \operatorname{Tr}\left(x_{b}\right) \neq 0 .\end{cases}
$$

where $B=(-1)^{\frac{p-1}{2} \frac{m-1}{2}} p^{\frac{m-3}{2}}$.

(II) If $m \equiv 2(\bmod 4)$, then we have the following.

- When $m_{p}=0$,

$$
N(b)=\left\{\begin{array}{lr}
-(p-1) p^{\frac{m}{2}-1}+p^{m-2} & \text { if } \operatorname{Tr}\left(x_{b}^{p+1}\right)=0 \text { and } \operatorname{Tr}\left(x_{b}\right)=0, \\
p^{m-2} & \text { if } \operatorname{Tr}\left(x_{b}^{p+1}\right)=0 \text { and } \operatorname{Tr}\left(x_{b}\right) \neq 0, \\
& \text { or } \operatorname{Tr}\left(x_{b}^{p+1}\right) \neq 0 \text { and } \operatorname{Tr}\left(x_{b}\right)=0, \\
-p^{\frac{m}{2}-1}+p^{m-2} & \text { if } \operatorname{Tr}\left(x_{b}^{p+1}\right) \neq 0 \text { and } \operatorname{Tr}\left(x_{b}\right) \neq 0 .
\end{array}\right.
$$

- When $m_{p} \neq 0$,

$$
N(b)=\left\{\begin{array}{lr}
p^{\frac{m}{2}-1}+p^{m-2} & \text { if } \operatorname{Tr}\left(x_{b}^{p+1}\right)=0 \text { and } \operatorname{Tr}\left(x_{b}\right)=0, \\
p^{m-2} & \text { if } \operatorname{Tr}\left(x_{b}^{p+1}\right)=0 \text { and } \operatorname{Tr}\left(x_{b}\right) \neq 0, \\
& \text { or } \operatorname{Tr}\left(x_{b}^{p+1}\right) \neq 0 \text { and } A=0, \\
p^{\frac{m}{2}-1}+p^{m-2} \text { or }-p^{\frac{m}{2}-1}+p^{m-2} & \text { if } \operatorname{Tr}\left(x_{b}^{p+1}\right) \neq 0 \text { and } A \neq 0,
\end{array}\right.
$$

where $A=-\frac{m_{p}}{4}+\frac{\operatorname{Tr}\left(x_{b}\right)^{2}}{4 \operatorname{Tr}\left(x_{b}^{p+1}\right)}$ and $x_{b}$ is the unique solution of the equation $x^{p^{2}}+x=-b^{p}$.

(III) If $m \equiv 0(\bmod 4)$, then we have the following.

- When $m_{p}=0$,

$$
N(b)= \begin{cases}-(p-1) p^{\frac{m}{2}-1}+p^{m-2} & \text { if } b \notin \operatorname{Im}(L), \\ -(p-1) p^{\frac{m}{2}}+p^{m-2} & \text { if } b \in \operatorname{Im}(L), \operatorname{Tr}\left(x_{b}^{p+1}\right)=0 \text { and } \operatorname{Tr}\left(x_{b}\right)=0, \\ p^{m-2} & \text { if } b \in \operatorname{Im}(L), \operatorname{Tr}\left(x_{b}^{p+1}\right)=0 \text { and } \operatorname{Tr}\left(x_{b}\right) \neq 0, \\ -p^{\frac{m}{2}}+p^{m-2} & \text { or } \operatorname{Tr}\left(x_{b}^{p+1}\right) \neq 0 \text { and } \operatorname{Tr}\left(x_{b}\right)=0,\end{cases}
$$

- When $m_{p} \neq 0$,

$$
N(b)= \begin{cases}p^{\frac{m}{2}-1}+p^{m-2} & \text { if } b \notin \operatorname{Im}(L), \\ p^{\frac{m}{2}}+p^{m-2} & \text { if } b \in \operatorname{Im}(L), \operatorname{Tr}\left(x_{b}^{p+1}\right)=0 \text { and } \operatorname{Tr}\left(x_{b}\right)=0, \\ p^{m-2} & \text { if } b \in \operatorname{Im}(L), \operatorname{Tr}\left(x_{b}^{p+1}\right)=0 \text { and } \operatorname{Tr}\left(x_{b}\right) \neq 0, \\ & \text { or } \operatorname{Tr}\left(x_{b}^{p+1} \neq 0 \text { and } A=0,\right. \\ p^{\frac{m}{2}}+p^{m-2} \text { or }-p^{\frac{m}{2}}+p^{m-2} & \text { if } b \in \operatorname{Im}(L), \operatorname{Tr}\left(x_{b}^{p+1}\right) \neq 0 \text { and } A \neq 0 .\end{cases}
$$


where $A=-\frac{m_{p}}{4}+\frac{\operatorname{Tr}\left(x_{b}\right)^{2}}{4 \operatorname{Tr}\left(x_{b}^{p+1}\right)}$ and $x_{b}$ is some solution of the equation $L(x)=x^{p^{2}}+x=-b^{p}$ when $b \in \operatorname{Im}(L)$.

Proof By definition, we have

$$
\begin{aligned}
N(b)= & p^{-2} \sum_{x \in \mathrm{GF}(q)}\left(\sum_{y \in \mathrm{GF}(p)} \zeta_{p}^{y \operatorname{Tr}\left(x^{p+1}-x\right)}\right)\left(\sum_{z \in \mathrm{GF}(p)} \zeta_{p}^{z \operatorname{Tr}(b x)}\right) \\
= & p^{-2} \sum_{z \in \mathrm{GF}(p)^{*}} \sum_{x \in \mathrm{GF}(q)} \zeta_{p}^{\operatorname{Tr}(b z x)}+p^{-2} \sum_{y \in \mathrm{GF}(p)^{*} x \in \operatorname{GF}(q)} \zeta_{p}^{\operatorname{Tr}\left(y x^{p+1}-y x\right)}+ \\
& p^{-2} \sum_{y \in \mathrm{GF}(p)^{*}} \sum_{z \in \mathrm{GF}(p)^{*}} \sum_{x \in \mathrm{GF}(q)} \zeta_{p}^{\operatorname{Tr}\left(y x^{p+1}+(b z-y) x\right)}+p^{m-2} .
\end{aligned}
$$

Note that

$$
\sum_{z \in \mathrm{GF}(p)^{*}} \sum_{x \in \mathrm{GF}(q)} \zeta_{p}^{\operatorname{Tr}(b z x)}=0
$$

The desired conclusions then follow from Lemmas 7 and 10 and the fact that

$$
(-1)^{\frac{p-1}{2}}{\sqrt{p^{*}}}^{m+1}=(-1)^{\frac{p-1}{2} \frac{m-1}{2}} p^{\frac{m+1}{2}} .
$$

In order to calculate the frequency of each weight in $\mathcal{C}_{D}$, we need a few more auxiliary results which are given and proved in the following three lemmas.

Lemma 12 For any $a \in \mathrm{GF}(p)$, let

$$
N_{a}=\left|\left\{x \in \operatorname{GF}(q): \operatorname{Tr}\left(x^{p+1}\right)=a\right\}\right| .
$$

Then

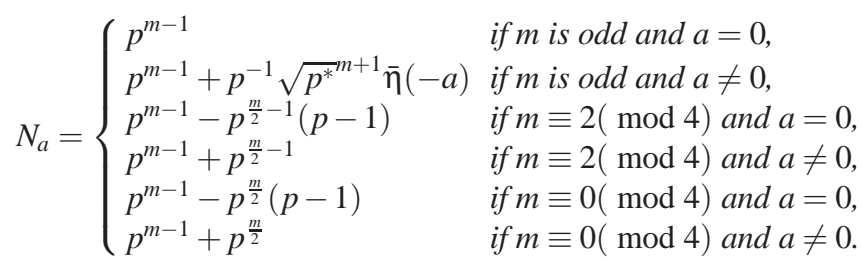


Proof By definition, Lemmas 4 and 5 we have

$$
\begin{aligned}
& N_{a}=\frac{1}{p} \sum_{x \in \mathrm{GF}(q)} \sum_{y \in \mathrm{GF}(p)} \zeta_{p}^{y\left(\operatorname{Tr}\left(x^{p+1}\right)-a\right)} \\
& =p^{m-1}+\frac{1}{p} \sum_{y \in \mathrm{GF}(p)^{*}} \zeta_{p}^{-y a} \sum_{x \in \mathrm{GF}(q)} \zeta_{p}^{\operatorname{Tr}\left(y x^{p+1}\right)} \\
& =p^{m-1}+\frac{1}{p} \sum_{y \in \mathrm{GF}(p)^{*}} \zeta_{p}^{-y a} S(y, 0) \\
& = \begin{cases}p^{m-1}+p^{-1} \sum_{y \in \operatorname{GF}(p)^{*}} \zeta_{p}^{-y a} \sqrt{p^{*}} \eta(y) & \text { if } m \text { is odd } \\
p^{m-1}+p^{-1} \sum_{y \in \operatorname{GF}(p)^{*}} \zeta_{p}^{-y a}\left(-p^{\frac{m}{2}}\right) & \text { if } m \equiv 2(\bmod 4) \\
p^{m-1}+p^{-1} \sum_{y \in \operatorname{GF}(p)^{*}} \zeta_{p}^{-y a}\left(-p^{\frac{m}{2}+1}\right) & \text { if } m \equiv 0(\bmod 4)\end{cases} \\
& = \begin{cases}p^{m-1}+p^{-1} \sqrt{p^{*}} \sum_{y \in \mathrm{GF}(p)^{*}} \bar{\eta}(y) & \text { if } m \text { is odd and } a=0 \\
p^{m-1}+p^{-1} \sqrt{p^{*}} \bar{\eta}(-a) \sum_{y \in \mathrm{GF}(p)^{*}} \zeta_{p}^{-y a} \bar{\eta}(-y a) & \text { if } m \text { is odd and } a \neq 0 \\
p^{m-1}+p^{-1}\left(-p^{\frac{m}{2}}\right) \sum_{y \in \mathrm{GF}(p)^{*}} \zeta_{p}^{-y a} & \text { if } m \equiv 2(\bmod 4) \\
p^{m-1}+p^{-1}\left(-p^{\frac{m}{2}+1}\right) \sum_{y \in \operatorname{GF}(p)^{*}} \zeta_{p}^{-y a} & \text { if } m \equiv 0(\bmod 4)\end{cases} \\
& = \begin{cases}p^{m-1}+p^{-1} \sqrt{p^{*}} \sum_{y \in \operatorname{GF}(p)^{*}} \bar{\eta}(y) & \text { if } m \text { is odd and } a=0, \\
p^{m-1}+p^{-1} \sqrt{p^{*}} \bar{\eta}(-a) G\left(\bar{\eta}, \bar{\chi}_{1}\right) & \text { if } m \text { is odd and } a \neq 0, \\
p^{m-1}-p^{\frac{m}{2}-1} \sum_{y \in \operatorname{GF}(p)^{*}} \zeta_{p}^{-y a} & \text { if } m \equiv 2(\bmod 4), \\
p^{m-1}-p^{\frac{m}{2}} \sum_{y \in \operatorname{GF}(p)^{*}} \zeta_{p}^{-y a} & \text { if } m \equiv 0(\bmod 4) .\end{cases}
\end{aligned}
$$

Note that $\sum_{y \in \operatorname{GF}(p)^{*}} \bar{\eta}(y)=0$ and

$$
\sum_{y \in \operatorname{GF}(p)^{*}} \zeta_{p}^{-y a}= \begin{cases}p-1 & \text { if } a=0, \\ -1 & \text { if } a \neq 0,\end{cases}
$$

The desired conclusion then follows from Lemma 1.

Lemma 13 For any $a \in \mathrm{GF}(p)$, let

$$
N_{(a, 0)}=\mid\left\{x \in \mathrm{GF}(q): \operatorname{Tr}\left(x^{p+1}\right)=a \text { and } \operatorname{Tr}(x)=0\right\} \mid .
$$

Then, for $m_{p}=0$, we have

$$
N_{(a, 0)}= \begin{cases}p^{m-2} & \text { if } m \text { is odd and } a=0, \\ p^{m-2}+p^{-1}{\sqrt{p^{*}}}^{m+1} \bar{\eta}(-a) & \text { if } m \text { is odd and } a \neq 0, \\ p^{m-2}-p^{\frac{m}{2}-1}(p-1) & \text { if } m \equiv 2(\bmod 4) \text { and } a=0, \\ p^{m-2}+p^{\frac{m}{2}-1} & \text { if } m \equiv 2(\bmod 4) \text { and } a \neq 0, \\ p^{m-2}-p^{\frac{m}{2}}(p-1) & \text { if } m \equiv 0(\bmod 4) \text { and } a=0, \\ p^{m-2}+p^{\frac{m}{2}} & \text { if } m \equiv 0(\bmod 4) \text { and } a \neq 0 .\end{cases}
$$

and for $m_{p} \neq 0$, we have

$$
N_{(a, 0)}= \begin{cases}p^{m-2}+p^{-2} \bar{\eta}\left(-m_{p}\right){\sqrt{p^{*}}}^{m+1}(p-1) & \text { if } m \text { is odd and } a=0, \\ p^{m-2}-p^{-2} \bar{\eta}\left(-m_{p}\right){\sqrt{p^{*}}}^{m+1} & \text { if } m \text { is odd and } a \neq 0, \\ p^{m-2} & \text { if } m \text { is even and } a=0, \\ p^{m-2}-\bar{\eta}\left(m_{p}\right) \bar{\eta}(a)(-1)^{\frac{p-1}{2}} p^{\frac{m}{2}-1} & \text { if } m \equiv 2(\bmod 4) \text { and } a \neq 0, \\ p^{m-2}-\bar{\eta}\left(m_{p}\right) \bar{\eta}(a)(-1)^{\frac{p-1}{2}} p^{\frac{m}{2}} & \text { if } m \equiv 0(\bmod 4) \text { and } a \neq 0 .\end{cases}
$$


Proof By definition, we have

$$
\begin{aligned}
N_{(a, 0)} & =p^{-2} \sum_{x \in \mathrm{GF}(q)}\left(\sum_{y \in \mathrm{GF}(p)} \zeta_{p}^{y\left(\operatorname{Tr}\left(x^{p+1}\right)-a\right)}\right)\left(\sum_{z \in \mathrm{GF}(p)} \zeta_{p}^{z \operatorname{Tr}(x)}\right) \\
& =p^{-2} \sum_{z \in \mathrm{GF}(p)} \sum_{x \in \mathrm{GF}(q)} \zeta_{p}^{\operatorname{Tr}(z x)}+p^{-2} \sum_{y \in \mathrm{GF}(p)^{*}} \sum_{z \in \mathrm{GF}(p)} \sum_{x \in \mathrm{GF}(q)} \zeta_{p}^{\operatorname{Tr}\left(y x^{p+1}+z x-y a\right)} \\
& =p^{-2}\left(q+\sum_{z \in \mathrm{GF}(p)^{*}} \sum_{x \in \mathrm{GF}(q)} \zeta_{p}^{\operatorname{Tr}(z x)}\right)+p^{-2} \sum_{y \in \mathrm{GF}(p)^{*}}\left(\zeta_{p}^{-y a} \sum_{z \in \mathrm{GF}(p)} \sum_{x \in \mathrm{GF}(q)} \zeta_{p}^{y\left(\operatorname{Tr}\left(x^{p+1}+\frac{z}{y} x\right)\right)}\right) .
\end{aligned}
$$

Note that

$$
\sum_{z \in \operatorname{GF}(p)^{*}} \sum_{x \in \operatorname{GF}(q)} \zeta_{p}^{\operatorname{Tr}(z x)}=0
$$

Therefore,

$$
\begin{aligned}
N_{(a, 0)} & =p^{m-2}+p^{-2} \sum_{y \in \mathrm{GF}(p)^{*}}\left(\zeta_{p}^{-y a} \sum_{z \in \mathrm{GF}(p)} \sum_{x \in \mathrm{GF}(q)} \zeta_{p}^{y\left(\operatorname{Tr}\left(x^{p+1}+z x\right)\right)}\right) \\
& =p^{m-2}+p^{-2} \sum_{y \in \mathrm{GF}(p)^{*}} \sigma_{y}\left(\zeta_{p}^{-a} \sum_{z \in \mathrm{GF}(p)} \sum_{x \in \mathrm{GF}(q)} \zeta_{p}^{\operatorname{Tr}\left(x^{p+1}+z x\right)}\right) .
\end{aligned}
$$

It is clear that $-\frac{1}{2} z$ is the solution of the equation $x^{p^{2}}+x=-z^{p}$ for any $z \in \operatorname{GF}(p)$. Hence, by Lemmas 4 and 5 , Equation 9 becomes

$$
\begin{aligned}
N_{(a, 0)}= & p^{m-2}+p^{-2} \sum_{y \in \mathrm{GF}(p)^{*}} \sigma_{y}\left(\zeta_{p}^{-a} \sum_{z \in \mathrm{GF}(p)} \sum_{x \in \mathrm{GF}(q)} \zeta_{p}^{\operatorname{Tr}\left(x^{p+1}+z x\right)}\right) \\
= & \begin{cases}p^{m-2}+p^{-2} \sum_{y \in \mathrm{GF}(p)^{*}} \sigma_{y}\left(\zeta_{p}^{-a} \sum_{z \in \mathrm{GF}(p)} \sqrt{p^{*}} \zeta_{p}^{-\frac{m_{p}}{4} z^{2}}\right) & \text { if } m \text { is odd } \\
p^{m-2}+p^{-2} \sum_{y \in \mathrm{GF}(p)^{*}} \sigma_{y}\left(\zeta_{p}^{-a} \sum_{z \in \mathrm{GF}(p)}-p^{\frac{m}{2}} \zeta_{p}^{-\frac{m_{p}}{4} z^{2}}\right) & \text { if } m \equiv 2(\bmod 4),(10) \\
p^{m-2}+p^{-2} \sum_{y \in \mathrm{GF}(p)^{*}} \sigma_{y}\left(\zeta_{p}^{-a} \sum_{z \in \mathrm{GF}(p)}-p^{\frac{m}{2}+1} \zeta_{p}^{-\frac{m_{p}}{4} z^{2}}\right) & \text { if } m \equiv 0(\bmod 4) .\end{cases}
\end{aligned}
$$

We distinguish the following two cases.

1. When $m_{p}=0$, Equation 10 becomes

$$
\begin{aligned}
N_{(a, 0)}= & \begin{cases}p^{m-2}+p^{-2} \sum_{y \in \mathrm{GF}(p)^{*}} \sigma_{y}\left(\zeta_{p}^{-a} \sum_{z \in \mathrm{GF}}(p){\sqrt{p^{*}}}^{m}\right) & \text { if } m \text { is odd, } \\
p^{m-2}+p^{-2} \sum_{y \in \mathrm{GF}(p)^{*}} \sigma_{y}\left(\zeta_{p}^{-a} \sum_{z \in \mathrm{GF}(p)}-p^{\frac{m}{2}}\right) & \text { if } m \equiv 2(\bmod 4), \\
p^{m-2}+p^{-2} \sum_{y \in \mathrm{GF}(p)^{*}} \sigma_{y}\left(\zeta_{p}^{-a} \sum_{z \in \mathrm{GF}(p)}-p^{\frac{m}{2}+1}\right) & \text { if } m \equiv 0(\bmod 4),\end{cases} \\
= & \begin{cases}p^{m-2}+p^{-1} \sqrt{p^{*}}{ }^{m-1} \sum_{y \in \mathrm{GF}(p)^{*}} \sigma_{y}\left(\zeta_{p}^{-a} \sqrt{p^{*}}\right) & \text { if } m \text { is odd, } \\
p^{m-2}-p^{\frac{m}{2}-1} \sum_{y \in \operatorname{GF}(p)^{*}} \sigma_{y}\left(\zeta_{p}^{-a}\right) & \text { if } m \equiv 2(\bmod 4), \\
p^{m-2}-p^{\frac{m}{2}} \sum_{y \in \mathrm{GF}(p)^{*}} \sigma_{y}\left(\zeta_{p}^{-a}\right) & \text { if } m \equiv 0(\bmod 4) .\end{cases}
\end{aligned}
$$

2. When $m_{p} \neq 0$, from Lemmas 2 and 1 we can easily get

$$
\sum_{z \in \mathrm{GF}(p)} \zeta_{p}^{-\frac{m_{p}}{4} z^{2}}=\bar{\eta}\left(-m_{p}\right) \sqrt{p^{*}}
$$


Thus, Equation 10 becomes

$$
\begin{aligned}
& N_{(a, 0)}= \begin{cases}p^{m-2}+p^{-2} \sum_{y \in \operatorname{GF}(p)^{*}} \sigma_{y}\left(\zeta_{p}^{-a} \bar{\eta}\left(-m_{p}\right){\sqrt{p^{*}}}^{m+1}\right) & \text { if } m \text { is odd, } \\
p^{m-2}+p^{-2} \sum_{y \in \operatorname{GF}(p)^{*}} \sigma_{y}\left(\zeta_{p}^{-a}\left(-p^{\frac{m}{2}}\right) \bar{\eta}\left(-m_{p}\right) \sqrt{p^{*}}\right) & \text { if } m \equiv 2(\bmod 4), \\
p^{m-2}+p^{-2} \sum_{y \in \operatorname{GF}(p)^{*}} \sigma_{y}\left(\zeta_{p}^{-a}\left(-p^{\frac{m}{2}+1}\right) \bar{\eta}\left(-m_{p}\right) \sqrt{p^{*}}\right) & \text { if } m \equiv 0(\bmod 4) .\end{cases} \\
&= \begin{cases}p^{m-2}+p^{-2} \bar{\eta}\left(-m_{p}\right){\sqrt{p^{*}}}^{m+1} \sum_{y \in \operatorname{GF}(p)^{*}} \sigma_{y}\left(\zeta_{p}^{-a}\right) & \text { if } m \text { is odd, } \\
p^{m-2}-p^{\frac{m}{2}-2} \bar{\eta}\left(-m_{p}\right) \sum_{y \in \operatorname{GF}(p)^{*}} \sigma_{y}\left(\zeta_{p}^{-a}{\sqrt{p^{*}}}^{m}\right. & \text { if } m \equiv 2(\bmod 4), \\
p^{m-2}-p^{\frac{m}{2}-1} \bar{\eta}\left(-m_{p}\right) \sum_{y \in \operatorname{GF}(p)^{*}} \sigma_{y}\left(\zeta_{p}^{-a}{\sqrt{p^{*}}}^{*}\right. & \text { if } m \equiv 0(\bmod 4) .\end{cases}
\end{aligned}
$$

The desired conclusions then follow from the facts that

$$
\sum_{y \in \mathrm{GF}(p)^{*}} \sigma_{y}\left(\zeta_{p}^{-a}\right)= \begin{cases}p-1 & \text { if } a=0 \\ -1 & \text { if } a \neq 0\end{cases}
$$

and

$$
\sum_{y \in \operatorname{GF}(p)^{*}} \sigma_{y}\left(\zeta_{p}^{-a} \sqrt{p^{*}}\right)= \begin{cases}0 & \text { if } a=0 \\ \left(\frac{-a}{p}\right) p^{*} & \text { if } a \neq 0\end{cases}
$$

Lemma 14 Suppose that $m_{p} \neq 0$. Let

$$
\bar{N}_{0}=\left|\left\{x \in \operatorname{GF}(q): \operatorname{Tr}\left(x^{p+1}\right)-\frac{1}{m_{p}} \operatorname{Tr}(x)^{2}=0\right\}\right| .
$$

Then

$$
\bar{N}_{0}= \begin{cases}p^{m-1}+p^{-1} \bar{\eta}\left(-m_{p}\right){\sqrt{p^{*}}}^{m+1}(p-1) & \text { if } m \text { is odd }, \\ p^{m-1} & \text { if } m \text { is even } .\end{cases}
$$

Proof By definition, we have

$$
\begin{aligned}
\bar{N}_{0} & =\frac{1}{p} \sum_{x \in \mathrm{GF}(q)} \sum_{y \in \mathrm{GF}(p)} \zeta_{p}^{y\left(\operatorname{Tr}\left(x^{p+1}\right)-\frac{1}{m_{p}} \operatorname{Tr}(x)^{2}\right)} \\
& =p^{m-1}+\frac{1}{p} \sum_{y \in \mathrm{GF}(p)^{*}} \sum_{x \in \mathrm{GF}(q)} \zeta_{p}^{y\left(\operatorname{Tr}\left(x^{p+1}\right)-\frac{1}{m_{p}} \operatorname{Tr}(x)^{2}\right)} \\
& =p^{m-1}+\frac{1}{p} \sum_{y \in \mathrm{GF}(p)^{*}} \sigma_{y}\left(\sum_{x \in \mathrm{GF}(q)} \zeta_{p}^{\operatorname{Tr}\left(x^{p+1}\right)-\frac{1}{m_{p}} \operatorname{Tr}(x)^{2}}\right) .
\end{aligned}
$$

Since the Fourier expansion of $\zeta_{p}^{-\frac{1}{m_{p}} X^{2}}$ can be expressed as

$$
\zeta_{p}^{-\frac{1}{m_{p}} X^{2}}=\sum_{z \in \mathrm{GF}(p)} a_{z} \zeta_{p}^{z X}
$$

for any $X \in \mathrm{GF}(p)$, we have

$$
a_{z}=\frac{1}{p} \sum_{X \in \mathrm{GF}(p)} \zeta_{p}^{-\frac{1}{m_{p}} X^{2}-z X}
$$

for any $z \in \mathrm{GF}(p)$ and

$$
\zeta_{p}^{-\frac{1}{m_{p}} \operatorname{Tr}(x)^{2}}=\sum_{z \in \operatorname{GF}(p)} a_{z} \zeta_{p}^{z \operatorname{Tr}(x)}
$$


Therefore, Equation 111 becomes

$$
\begin{aligned}
\bar{N}_{0}= & p^{m-1}+\frac{1}{p} \sum_{y \in \mathrm{GF}(p)^{*}} \sigma_{y}\left(\sum_{z \in \mathrm{GF}(p)} a_{z} \sum_{x \in \mathrm{GF}(q)} \zeta_{p}^{\operatorname{Tr}\left(x^{p+1}\right)+z \operatorname{Tr}(x)}\right) \\
= & \begin{cases}p^{m-1}+p^{-1} \sum_{y \in \mathrm{GF}(p)^{*}} \sigma_{y}\left({\sqrt{p^{*}}}^{m} \sum_{z \in \mathrm{GF}(p)} a_{z} \zeta_{p}^{-\frac{m_{p}}{4} z^{2}}\right) & \text { if } m \text { is odd } \\
p^{m-1}+p^{-1} \sum_{y \in \mathrm{GF}(p)^{*}} \sigma_{y}\left(-p^{\frac{m}{2}} \sum_{z \in \mathrm{GF}(p)} a_{z} \zeta_{p}^{-\frac{m_{p}}{4} z^{2}}\right) & \text { if } m \equiv 2(\bmod 4), \\
p^{m-1}+p^{-1} \sum_{y \in \mathrm{GF}(p)^{*}} \sigma_{y}\left(-p^{\frac{m+1}{2}} \sum_{z \in \mathrm{GF}(p)} a_{z} \zeta_{p}^{-\frac{m_{p}}{4} z^{2}}\right) & \text { if } m \equiv 0(\bmod 4) .\end{cases}
\end{aligned}
$$

However, by Equation (12) and Lemma 2 we obtain

$$
\begin{aligned}
\sum_{z \in \mathrm{GF}(p)} a_{z} \zeta_{p}^{-\frac{m_{p}}{4} z^{2}} & =\sum_{z \in \mathrm{GF}(p)} \frac{1}{p} \sum_{X \in \mathrm{GF}(p)} \zeta_{p}^{-\frac{1}{m_{p}} X^{2}-z X-\frac{m_{p}}{4} z^{2}} \\
& =\sum_{z \in \mathrm{GF}(p)} \frac{1}{p} \bar{\eta}\left(-\frac{1}{m_{p}}\right) G\left(\bar{\eta}, \bar{\chi}_{1}\right) \\
& =\bar{\eta}\left(-m_{p}\right) \sqrt{p^{*}} .
\end{aligned}
$$

Hence, Equation (13) becomes

$$
\bar{N}_{0}= \begin{cases}p^{m-1}+p^{-1}{\sqrt{p^{*}}}^{m+1} \bar{\eta}\left(-m_{p}\right) \sum_{y \in \mathrm{GF}(p)^{*}} \sigma_{y}(1) & \text { if } m \text { is odd } \\ p^{m-1}+p^{-1}\left(-p^{\frac{m}{2}}\right) \bar{\eta}\left(-m_{p}\right) \sum_{y \in \mathrm{GF}(p)^{*}} \sigma_{y}\left(\sqrt{p^{*}}\right) & \text { if } m \equiv 2(\bmod 4), \\ p^{m-1}+p^{-1}\left(-p^{\frac{m+1}{2}}\right) \bar{\eta}\left(-m_{p}\right) \sum_{y \in \mathrm{GF}(p)^{*}} \sigma_{y}\left(\sqrt{p^{*}}\right) & \text { if } m \equiv 0(\bmod 4)\end{cases}
$$

The desired conclusions then follow from the fact that

$$
\left\{\begin{array}{l}
\sigma_{y}(1)=1 \\
\sum_{y \in \operatorname{GF}(p)^{*}} \sigma_{y}\left(\sqrt{p^{*}}\right)=0
\end{array}\right.
$$

4.2 The proof of Theorems 1,2 and 3

By definition, the code length of $\mathcal{C}_{D}$ is $n=|D|=n_{0}-1$, where $n_{0}$ was defined in Lemma 8 . This means that Equations (6) and (7) follow.

For each $b \in \mathrm{GF}(q)^{*}$, define

$$
\mathbf{c}_{b}=\left(\operatorname{Tr}\left(b d_{1}\right), \operatorname{Tr}\left(b d_{2}\right), \ldots, \operatorname{Tr}\left(b d_{n}\right)\right),
$$

where $d_{1}, d_{2}, \ldots, d_{n}$ are the elements of $D$. Then the Hamming weight $\operatorname{wt}\left(\mathbf{c}_{b}\right)$ of $\mathbf{c}_{b}$ is

$$
\mathrm{wt}\left(\mathbf{c}_{b}\right)=n_{0}-N(b),
$$

where $n_{0}$ and $N(b)$ were defined before. By Lemmas 8 and 11 we have wt $\left(\mathbf{c}_{b}\right)=n_{0}-N(b)>$ 0 for each $b \in \operatorname{GF}(q)^{*}$. This means that the code $\mathcal{C}_{D}$ has $q$ distinct codewords. Hence, the dimension of the code $\mathcal{C}_{D}$ is $m$.

Next we shall prove the multiplicities $A_{w_{i}}$ of the codewords with weight $w_{i}$ in $\mathcal{C}_{D}$, and will distinguish the following three cases.

1. The case that $m$ is odd.

It follows from Lemma 4 that $L(x)=x^{p^{2}}+x$ is a permutation polynomial over $\operatorname{GF}(q)$. Thus the unique solution $x_{b}$ of $L(x)=-b^{p}$ runs through $\operatorname{GF}(q)^{*}$ when $b$ runs through $\mathrm{GF}(q)^{*}$. 
- When $m_{p}=0$, the desired conclusion of Table 1 follows from Equation (15) and Lemmas 811,12 and 13 .

- When $m_{p} \neq 0$, we only give the proof for the case $\bar{\eta}\left(m_{p}\right)=1$ and omit the proof for the case $\bar{\eta}\left(m_{p}\right)=-1$ whose proof is similar. Suppose that $m_{2}=1$ and $\bar{\eta}\left(m_{p}\right)=1$, then from Lemmas 8 and 11 we obtain

$$
\begin{aligned}
\operatorname{wt}\left(\mathbf{c}_{b}\right)= & n_{0}-N(b) \\
= & \begin{array}{ll}
B_{1} & \text { if } \operatorname{Tr}\left(x_{b}^{p+1}\right)=0 \text { and } \operatorname{Tr}\left(x_{b}\right)=0, \\
B_{1}+B p & \text { if } \operatorname{Tr}\left(x_{b}^{p+1}\right)=0 \text { and } \operatorname{Tr}\left(x_{b}\right) \neq 0, \\
B_{1}+B(p+1) & \text { if } \operatorname{Tr}\left(x_{b}^{p+1}\right) \in \mathrm{SQ} \text { and } \operatorname{Tr}\left(x_{b}\right)=0, \\
B_{1}+B(p-1) & \text { if } \operatorname{Tr}\left(x_{b}^{p+1}\right) \in \mathrm{NSQ}, \\
B_{1}+B \text { or } B_{1}+B(p+1) & \text { if } \operatorname{Tr}\left(x_{b}^{p+1}\right) \in \mathrm{SQ} \text { and } \operatorname{Tr}\left(x_{b}\right) \neq 0,
\end{array}
\end{aligned}
$$

where $B_{1}=p^{m-2}(p-1)$ and $B=(-1)^{\frac{p-1}{2} \frac{m-1}{2}} p^{\frac{m-3}{2}}$. Therefore, the weight $w \mathrm{t}\left(\mathbf{c}_{b}\right)$ satisfies

$$
\operatorname{wt}\left(\mathbf{c}_{b}\right) \in\left\{B_{1}, B_{1}+B p, B_{1}+B(p+1), B_{1}+B(p-1), B_{1}+B\right\}
$$

for each $b \in \mathrm{GF}(q)^{*}$. Define

$$
\begin{aligned}
& w_{1}=B_{1}, \\
& w_{2}=B_{1}+B p, \\
& w_{3}=B_{1}+B(p+1), \\
& w_{4}=B_{1}+B(p-1), \\
& w_{5}=B_{1}+B .
\end{aligned}
$$

We now determine the number $A_{w_{i}}$ of the codewords with weight $w_{i}$ in $\mathcal{C}_{D}$. By Lemmas 12 and 13 we can directly determine

$$
\left\{\begin{array}{l}
A_{w_{1}}=N_{(0,0)}-1=p^{m-2}-1+(p-1) B, \\
A_{w_{2}}=\left(N_{0}-1\right)-\left(N_{(0,0)}-1\right)=B_{1}-(p-1) B, \\
A_{w_{4}}=\frac{p-1}{2} N_{a}=\frac{p-1}{2}\left(p^{m-1}-B p\right),
\end{array}\right.
$$

where $a$ is a nonsquare in $\operatorname{GF}(p)^{*}$. Since $0 \notin D$, the minimum distance $d^{\perp}$ of the dual code $\mathcal{C}_{D}^{\perp}$ of $\mathcal{C}_{D}$ cannot be 1 . This means that the minimum weight of the dual code $C_{D}^{\perp}$ is at least 2. The first two Pless Power Moments [16, p.260] lead to the following system of equations:

$$
\begin{cases}A_{w_{1}}+A_{w_{2}}+A_{w_{3}}+A_{w_{4}}+A_{w_{5}} & =p^{m}-1 \\ w_{1} A_{w_{1}}+w_{2} A_{w_{2}}+w_{3} A_{w_{3}}+w_{4} A_{w_{4}}+w_{5} A_{w_{5}} & =(p-1) n p^{m-1}\end{cases}
$$

where the code length $n=n_{0}-1=p^{m-1}-1+p B$. Solving the system of equations in 17 yields

$$
\left\{\begin{array}{l}
A_{w_{3}}=\frac{1}{2}(p-1)(p-2) p^{\frac{m-3}{2}}\left(p^{\frac{m-1}{2}}-(-1)^{\frac{p-1}{2} \frac{m-1}{2}}\right) \\
A_{w_{5}}=(p-1) p^{m-2}+(-1)^{\frac{p-1}{2} \frac{m-1}{2}}(p-1)^{2} p^{\frac{m-3}{2}}
\end{array}\right.
$$

This completes the proof of the weight distribution of Table 2 
2. The case that $m \equiv 2(\bmod 4)$.

It follows from Lemma 5 that $L(x)=x^{p^{2}}+x$ is a permutation polynomial over $\operatorname{GF}(q)$. Similar to the analysis for the case $m$ being odd, we have the following.

- When $m_{p}=0$, it is clear that the desired conclusion of Table 3 follows from Equation 15) and Lemmas $8,11,12$ and 13

- When $m_{p} \neq 0$, by Lemmas 8 and 11 we have

$\mathrm{wt}\left(\mathbf{c}_{b}\right)=n_{0}-N(b)$

$$
=\left\{\begin{array}{lr}
(p-1) p^{m-2} & \text { if } \operatorname{Tr}\left(x_{b}^{p+1}\right)=0 \text { and } \operatorname{Tr}\left(x_{b}\right)=0, \\
(p-1) p^{m-2}+p^{\frac{m}{2}-1} & \text { if } \operatorname{Tr}\left(x_{b}^{p+1}\right)=0 \text { and } \operatorname{Tr}\left(x_{b}\right) \neq 0 \\
& \text { or } \operatorname{Tr}\left(x_{b}^{p+1}\right) \neq 0 \text { and } A=0, \\
(p-1) p^{m-2} \text { or }(p-1) p^{m-2}+2 p^{\frac{m}{2}-1} & \text { if } \operatorname{Tr}\left(x_{b}^{p+1}\right) \neq 0 \text { and } A \neq 0,
\end{array}\right.
$$

where $A=-\frac{m_{p}}{4}+\frac{\operatorname{Tr}\left(x_{b}\right)^{2}}{4 \operatorname{Tr}\left(x_{b}^{p+1}\right)}$. Therefore, the weight wt $\left(\mathbf{c}_{b}\right)$ satisfies

$$
\operatorname{wt}\left(\mathbf{c}_{b}\right) \in\left\{(p-1) p^{m-2},(p-1) p^{m-2}+p^{\frac{m}{2}-1},(p-1) p^{m-2}+2 p^{\frac{m}{2}-1}\right\}
$$

for each $b \in \operatorname{GF}(q)^{*}$. Define

$$
\begin{aligned}
& w_{1}=(p-1) p^{m-2}, \\
& w_{2}=(p-1) p^{m-2}+p^{\frac{m}{2}-1}, \\
& w_{3}=(p-1) p^{m-2}+2 p^{\frac{m}{2}-1} .
\end{aligned}
$$

We now determine the number $A_{w_{i}}$ of the codewords with weight $w_{i}$ in $\mathcal{C}_{D}$. Note that

$$
\begin{aligned}
& \mid\left\{b \in \operatorname{GF}(q): \operatorname{Tr}\left(x_{b}^{p+1}\right) \neq 0 \text { and } A=0\right\} \mid \\
& =|\{b \in \operatorname{GF}(q): A=0\}|-\mid\left\{b \in \operatorname{GF}(q): \operatorname{Tr}\left(x_{b}^{p+1}\right)=0 \text { and } A \neq 0\right\} \mid \\
& =\left|\left\{x \in \operatorname{GF}(q): \operatorname{Tr}\left(x^{p+1}\right)-\frac{1}{m_{p}} \operatorname{Tr}(x)^{2}=0\right\}\right|-\mid\left\{x \in \operatorname{GF}(q): \operatorname{Tr}\left(x^{p+1}\right)=0 \text { and } \operatorname{Tr}(x)=0\right\} \mid,
\end{aligned}
$$

as $L(x)=x^{p^{2}}+x$ is a permutation polynomial over $\operatorname{GF}(q)$ for this case $m \equiv 2$ $(\bmod 4)$. Thus from Equation (18) and Lemmas 12, 13 and 14, we can directly obtain

$$
A_{w_{2}}=\left(N_{0}-N_{(0,0)}\right)+\left(\bar{N}_{0}-N_{(0,0)}\right)=(p-1)\left(2 p^{m-2}-p^{\frac{m}{2}-1}\right) .
$$

Since the minimum weight of the dual code $\mathcal{C}_{D}^{\perp}$ is at least 2, from the first two Pless Power Moments [16, p.260] we can compute $A_{w_{1}}$ and $A_{w_{3}}$. This completes the proof of the weight distribution of Table 4

3. The case that $m \equiv 0(\bmod 4)$.

- If $m_{p}=0$, then by Lemmas 8 and 11 we have

$$
\begin{aligned}
\operatorname{wt}\left(\mathbf{c}_{b}\right)= & n_{0}-N(b) \\
= & \begin{cases}B_{1}-(p-1)^{2} p^{\frac{m}{2}-1} & \text { if } b \notin \operatorname{Im}(L), \\
B_{1} & \text { if } b \in \operatorname{Im}(L), \operatorname{Tr}\left(x_{b}^{p+1}\right)=0 \text { and } \operatorname{Tr}\left(x_{b}\right)=0, \\
B_{1}-(p-1) p^{\frac{m}{2}} & \text { if } b \in \operatorname{Im}(L), \operatorname{Tr}\left(x_{b}^{p+1}\right)=0 \text { and } \operatorname{Tr}\left(x_{b}\right) \neq 0 \\
& \text { or } \operatorname{Tr}\left(x_{b}^{p+1}\right) \neq 0 \text { and } \operatorname{Tr}\left(x_{b}\right)=0, \\
B_{1}-(p-2) p^{\frac{m}{2}} & \text { if } b \in \operatorname{Im}(L), \operatorname{Tr}\left(x_{b}^{p+1}\right) \neq 0 \text { and } \operatorname{Tr}\left(x_{b}\right) \neq 0,\end{cases}
\end{aligned}
$$

where $B_{1}=p^{m-2}(p-1)$.

Recall that $L(x)=x^{p^{2}}+x$. If $b \in \operatorname{Im}(L)$, then this means that $L(x)=-b^{p}$ is solvable. We point out the following facts: 
- If $m \equiv 0(\bmod 4)$, then $L(x)$ is not a permutation polynomial over $\operatorname{GF}(q)$. However, for any $b \in \operatorname{GF}(q)$, if $L(x)=-b^{p}$ is solvable in this case $m \equiv 0(\bmod 4)$, then it has $p^{2}$ solutions.

- If $m \equiv 0(\bmod 4)$, then the number of $b$ is $p^{m-2}$ such that $L(x)=-b^{p}$ is solvable when $b$ runs through $\operatorname{GF}(q)$.

Therefore, we have

$$
\operatorname{wt}\left(\mathbf{c}_{b}\right)= \begin{cases}p^{m-2}(p-1)-(p-1)^{2} p^{\frac{m}{2}-1} & \text { occurs } p^{m}-p^{m-2} \text { times, } \\ p^{m-2}(p-1) & \text { occurs } p^{-2} N_{(0,0)}-1 \text { times, } \\ (p-1) p^{\frac{m}{2}}\left(p^{\frac{m}{2}-2}-1\right) & \text { occurs }\left(N_{0}+p^{m-1}-2 N_{(0,0)}\right) p^{-2} \text { times, } \\ (p-1) p^{m-2}-(p-2) p^{\frac{m}{2}} & \text { occurs }\left(p^{m}-p^{m-1}-N_{0}+N_{(0,0)}\right) p^{-2} \text { times, }\end{cases}
$$

when $b$ runs through $\operatorname{GF}(q)^{*}$, where $N_{0}$ and $N_{(0,0)}$ were defined before. The desired conclusion of Table 5 then follows from Lemmas 12 and 13

- If $m_{p} \neq 0$, then from Lemmas 8 and 11 we get

$$
\begin{aligned}
\mathrm{wt}\left(\mathbf{c}_{b}\right)= & n_{0}-N(b) \\
= & \begin{cases}(p-1) p^{\frac{m}{2}-1}+B_{1} & \text { if } b \notin \operatorname{Im}(L), \\
B_{1} & \text { if } b \in \operatorname{Im}(L), \operatorname{Tr}\left(x_{b}^{p+1}\right)=0 \text { and } \operatorname{Tr}\left(x_{b}\right)=0, \\
B_{1}+p^{\frac{m}{2}} & \text { if } b \in \operatorname{Im}(L), \operatorname{Tr}\left(x_{b}^{p+1}\right)=0 \text { and } \operatorname{Tr}\left(x_{b}\right) \neq 0 \\
& \text { or } \operatorname{Tr}\left(x_{b}^{p+1}\right) \neq 0 \text { and } A=0, \\
B_{1} \text { or } B_{1}+2 p^{\frac{m}{2}} & \text { if } b \in \operatorname{Im}(L), \operatorname{Tr}\left(x_{b}^{p+1}\right) \neq 0 \text { and } A \neq 0,\end{cases}
\end{aligned}
$$

where $B_{1}=p^{m-2}(p-1)$. Therefore, the weight $\operatorname{wt}\left(\mathbf{c}_{b}\right)$ satisfies

$$
\operatorname{wt}\left(\mathbf{c}_{b}\right) \in\left\{(p-1) p^{\frac{m}{2}-1}+B_{1}, B_{1}, B_{1}+p^{\frac{m}{2}}, B_{1}+2 p^{\frac{m}{2}}\right\}
$$

for each $b \in \operatorname{GF}(q)^{*}$. Define

$$
\begin{aligned}
& w_{1}=(p-1) p^{\frac{m}{2}-1}+B_{1}, \\
& w_{2}=B_{1}, \\
& w_{3}=B_{1}+p^{\frac{m}{2}}, \\
& w_{4}=B_{1}+2 p^{\frac{m}{2}} .
\end{aligned}
$$

We now determine the number $A_{w_{i}}$ of the codewords with weight $w_{i}$ in $\mathcal{C}_{D}$. It is clear that

$$
A_{w_{1}}=p^{m}-p^{m-2} \text {. }
$$

By Equation 19, and Lemmas 12, 13, and 14, we can directly obtain

$$
\begin{aligned}
A_{w_{3}} & =p^{-2}\left(N_{0}-N_{(0,0)}+\bar{N}_{0}\right), \\
& =(p-1)\left(2 p^{m-4}-p^{\frac{m}{2}-2}\right) .
\end{aligned}
$$

Since the minimum weight of the dual code $\mathcal{C}_{D}^{\perp}$ is at least 2, the first two Pless Power Moments [16, p.260] lead to the following system of equations:

$$
\begin{cases}A_{w_{1}}+A_{w_{2}}+A_{w_{3}}+A_{w_{4}} & =p^{m}-1, \\ w_{1} A_{w_{1}}+w_{2} A_{w_{2}}+w_{3} A_{w_{3}}+w_{4} A_{w_{4}} & =(p-1) n p^{m-1}\end{cases}
$$

where $n=n_{0}-1=p^{m-1}+p^{\frac{m}{2}}-1$. Solving the system of equations in 201 gives

$$
\left\{\begin{array}{l}
A_{w_{2}}=p^{m-4}+\frac{p-1}{2}\left(p^{\frac{m}{2}-1}+p^{m-3}\right)-1 \\
A_{w_{4}}=\frac{1}{2}(p-1)(p-2)\left(p^{m-4}-p^{\frac{m}{2}-2}\right) .
\end{array}\right.
$$

This completes the proof of the weight distribution of Table 6 Summarizing all the conclusions above completes the proofs of Theorems 1, 2 and 3 


\section{Concluding remarks}

In this paper, we presented a class of linear codes $\mathcal{C}_{D}$ with a few weights and completely determined their weight distributions. The result showed that they have at most five weights. Particularly, the codes $C_{D}$ presented in this paper have three weights when $m \geq 6$ and $m \equiv$ $2(\bmod 4)$. Many classes of linear codes with a few weights were constructed (see, for example, [14, 29, 23, 2, 15, 18, 4, 28, 12]). When $m$ is odd, the code $\mathcal{C}_{D}$ presented in this paper has the same parameter and weight distribution as that of $\mathcal{C}_{D}$ presented in [19]. But they have different defining set $D$.

Any linear code over $\mathrm{GF}(p)$ can be used to obtain secret sharing schemes [4,27]. In order to obtain secret sharing schemes with interesting access structures, one would like to have linear codes $C$ such that $w_{\min } / w_{\max }>\frac{p-1}{p}$ [27], where $w_{\min }$ and $w_{\max }$ denote the minimum and maximum nonzero weight of the linear code.

When $m \geq 5$ is odd and $m_{p}=0$, the code $C_{D}$ of Section 3 satisfies that

$$
\frac{w_{\min }}{w_{\max }}=\frac{p^{m-2}-p^{(m-3) / 2}}{p^{m-2}+p^{(m-3) / 2}}>\frac{p-1}{p} .
$$

When $m \geq 5$ is odd and $m_{p} \neq 0$, the code $\mathcal{C}_{D}$ of Section 3 satisfies that

$$
\frac{w_{\min }}{w_{\max }}=\frac{(p-1) p^{m-2}-(p+1) p^{(m-3) / 2}}{(p-1) p^{m-2}+(p+1) p^{(m-3) / 2}}>\frac{p-1}{p} .
$$

When $m \geq 6, m \equiv 2(\bmod 4)$ and $m_{p}=0$, the code $\mathcal{C}_{D}$ of Section 3 satisfies that

$$
\frac{w_{\min }}{w_{\max }}=\frac{p^{m-2}-p^{m / 2-1}}{p^{m-2}}>\frac{p-1}{p} .
$$

When $m \geq 6, m \equiv 2(\bmod 4)$ and $m_{p} \neq 0$, the code $\mathcal{C}_{D}$ of Section 3 satisfies that

$$
\frac{w_{\min }}{w_{\max }}=\frac{(p-1) p^{m-2}}{(p-1) p^{m-2}+2 p^{m / 2-1}}>\frac{p-1}{p} .
$$

When $m \geq 6, m \equiv 0(\bmod 4)$ and $m_{p}=0$, the code $\mathcal{C}_{D}$ of Section 3 satisfies that

$$
\frac{w_{\min }}{w_{\max }}=\frac{p^{m-2}-p^{m / 2}}{p^{m-2}}>\frac{p-1}{p} .
$$

When $m \geq 6, m \equiv 0(\bmod 4)$ and $m_{p} \neq 0$, the code $\mathcal{C}_{D}$ of Section 3 satisfies that

$$
\frac{w_{\min }}{w_{\max }}=\frac{(p-1) p^{m-2}}{(p-1) p^{m-2}+2 p^{m / 2}}>\frac{p-1}{p} .
$$

Hence, the linear codes $\mathcal{C}_{D}$ presented in this paper satisfy the condition that $w_{\min } / w_{\max }>$ $\frac{p-1}{p}$, and can be employed to obtain secret sharing schemes with interesting access structures $104,14,27$. 


\section{References}

1. Anderson, R., Ding, C., Helleseth, T., Kløve, T.: How to build robust shared control systems, Des. Codes Cryptogr. 15(2), 111-124 (1998)

2. Calderbank, A. R., Goethals, J. M.: Three-weight codes and association schemes, Philips J. Res. 39, 143-152 (1984)

3. Calderbank, A. R., Kantor, W. M.: The geometry of two-weight codes, Bull. London Math. Soc. 18, 97-122 (1986)

4. Carlet, C., Ding, C., Yuan, J.: Linear codes from perfect nonlinear mappings and their secret sharing schemes, IEEE Trans. Inf. Theory 51(6), 2089-2102 (2005)

5. Coulter, R.S.: Further evaluations of Weil sums, Acta Arith. 86(3), 217-226 (1998)

6. Ding, C.: Linear codes from some 2-designs, IEEE Trans. Inf. Theory 60(6), 3265-3275 (2015)

7. Ding, C.: A class of three-weight and four-weight codes, in: Xing C. et al. (Eds.), Proc. of the Second International Workshop on Coding Theory and Cryptography, Lecture Notes in Computer Science, vol. 5557, pp. 34-42. Springer Verlag, Berlin (2009)

8. Ding, C.: A Construction of Binary Linear Codes from Boolean Functions, arXiv:1511.00321 (2015)

9. Ding, C., Luo, J., Niederreiter, H.: Two weight codes punctured from irreducible cyclic codes, in: Y. Li, S. Ling, H. Niederreiter, H. Wang, C. Xing, S. Zhang (Eds.), Proc. of the First International Workshop on Coding Theory and Cryptography, pp. 119-124. World Scientific, Singapore (2008)

10. Ding, C., Niederreiter, H.: Cyclotomic linear codes of order 3, IEEE Trans. Inf. Theory 53(6), 2274-2277 (2007)

11. Ding, C., Wang, X.: A coding theory construction of new systematic authentication codes, Theoretical Computer Science 330, 81-99 (2005)

12. Ding, C., Li, C., Li, N., Zhou, Z.: Three-weight cyclic codes and their weight distributions, Discrete Mathematics 339(2), 415-427 (2014)

13. Ding, K., Ding, C.: Binary linear codes with three weights, IEEE Communication Letters 18(11), 1879$1882(2014)$

14. Ding, K., Ding, C.: A class of two-weight and three-weight codes and their applications in secret sharing, IEEE Trans. Inf. Theory 61(11), 5835-5842 (2015)

15. Feng, K., Luo, J.: Value distribution of exponential sums from perfect nonlinear functions and their applications, IEEE Trans. Inform. Theory 53(9), 3035-3041(2007)

16. Huffman, W. C., Pless, V.: Fundamentals of Error-Correcting Codes. Cambridge University Press, Cambridge (2003)

17. K. Ireland, M. Rosen, A Classical Introduction to Modern Number Theory, 2nd ed. New York: SpringerVerlag, 1990, vol. 84, Graduate Texts in Mathematics.

18. Li, C., Yue, Q., Li, F.: Weight distributions of cyclic codes with respect to pairwise coprime order elements, Finite Fields and Their Applications 28, 94-114 (2014)

19. Li, F., Wang, Q., Lin, D.: A class of three-weight and five-weight linear codes, arXiv:1509.06242 1 , (2015)

20. Lidl, R., Niederreiter, H.: Finite Fields. Cambridge University Press, Cambridge (1997)

21. Mesnager, S.: Linear codes with few weights from weakly regular bent functions based on a generic construction, IACR Cryptology ePrint Archive 2015: 1103.

22. Qi, Y., Tang, C., Huang, D.: Binary linear codes with few weights, to appear IEEE Communications Letters, (2015)

23. Tang, C., Li, N., Qi, Y., Zhou, Z., Helleseth, T.: Linear codes with two or three weights from weakly regular bent functions, to appear IEEE Trans. Inform. Theory, (2015)

24. Tang, C., Qi, Y., Huang, D.: Two-weight and three-weight linear codes from square functions, to appear IEEE Communications Letters, (2015)

25. Wang, Q., Ding, K., Xue, R.: Binary linear codes with two weights, IEEE Communications Letters 19, 1097-1100 (2015).

26. Xiang, C.: Linear codes from a generic construction, Cryptography and Communications, http://link.springer.com/article/10.1007/s12095-015-0158-1 (2015)

27. Yuan, J., Ding, C.: Secret sharing schemes from three classes of linear codes, IEEE Trans. Inf. Theory 52(1), 206-212 (2006)

28. Zhou, Z., Ding, C.: A class of three-weight cyclic codes, Finite Fields Appl 25, 79-93 (2014)

29. Zhou, Z., Li, N., Fan, C., Helleseth, T.: Linear codes with two or three weights from quadratic bent functions, Des. Codes Cryptogr, DOI 10.1007/s10623-015-0144-9, (2015) 